\title{
ON THE DEARTH OF COMPACT, MASSIVE, RED SEQUENCE GALAXIES IN THE LOCAL UNIVERSE
}

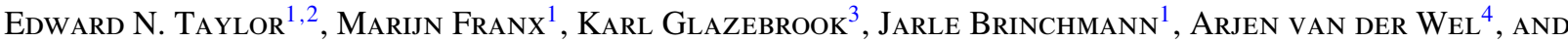 \\ PIETER G VAN DOKKUM ${ }^{5}$ \\ ${ }^{1}$ Sterrewacht Leiden, Leiden University, NL-2300 RA Leiden, The Netherlands; ent@ strw.leidenuniv.nl \\ ${ }^{2}$ School of Physics, The University of Melbourne, Parkville 3010, Australia \\ ${ }^{3}$ Centre for Astrophysics \& Supercomputing, Swinburne University of Technology, Hawthorn 3122, Australia \\ ${ }^{4}$ Max Planck Institut für Astronomie, D-69117 Heidelberg, Germany \\ ${ }^{5}$ Department of Astronomy, Yale University, New Haven, CT 06520-8101, USA \\ Received 2009 July 23; accepted 2010 April 6; published 2010 August 13
}

\begin{abstract}
We set out to test the claim that the recently identified population of compact, massive, and quiescent galaxies at $z \sim 2.3$ must undergo significant size evolution to match the properties of galaxies found in the local universe. Using data from the Sloan Digital Sky Survey (SDSS; Data Release 7), we have conducted a search for local red sequence galaxies with sizes and masses comparable to those found at $z \sim 2.3$. The SDSS spectroscopic target selection algorithm excludes high surface brightness objects; we show that this makes incompleteness a concern for such massive, compact galaxies, particularly for low redshifts $(z \lesssim 0.05)$. We have identified $63 M_{*}>10^{10.7} M_{\odot}$ $\left(\approx 5 \times 10^{10} M_{\odot}\right)$ red sequence galaxies at $0.066<z_{\text {spec }}<0.12$ which are smaller than the median size-mass relation by a factor of 2 or more. Consistent with expectations from the virial theorem, the median offset from the mass-velocity dispersion relation for these galaxies is 0.12 dex. We do not, however, find any galaxies with sizes and masses comparable to those observed at $z \sim 2.3$, implying a decrease in the comoving number density of these galaxies, at fixed size and mass, by a factor of $\gtrsim 5000$. This result cannot be explained by incompleteness: in the $0.066<z<0.12$ interval, we estimate that the SDSS spectroscopic sample should typically be $\gtrsim 75 \%$ complete for galaxies with the sizes and masses seen at high redshift, although for the very smallest galaxies it may be as low as $\sim 20 \%$. In order to confirm that the absence of such compact massive galaxies in SDSS is not produced by spectroscopic selection effects, we have also looked for such galaxies in the basic SDSS photometric catalog, using photometric redshifts. While we do find signs of a slight bias against massive, compact galaxies, this analysis suggests that the SDSS spectroscopic sample is missing at most a few objects in the regime we consider. Accepting the high-redshift results, it is clear that massive galaxies must undergo significant structural evolution over $z \lesssim 2$ in order to match the population seen in the local universe. Our results suggest that a highly stochastic mechanism (e.g., major mergers) cannot be the primary driver of this strong size evolution.
\end{abstract}

Key words: galaxies: evolution - galaxies: formation - galaxies: fundamental parameters

Online-only material: color figures, machine-readable table

\section{INTRODUCTION}

In the simplest possible terms, the naive expectation from hierarchical structure formation scenarios is that the most massive galaxies form relatively late. This is in contrast to the observation that the bulk of cosmic star formation occurs in galaxies with progressively lower stellar masses at later times (e.g., Juneau et al. 2005; Zheng et al. 2007; Damen et al. 2008), the so-called downsizing of galaxy growth. These observations have been accommodated within the $\Lambda$ CDM framework with the introduction of a quenching mechanism (e.g., Menci et al. 2005; Croton et al. 2006; Cattaneo et al. 2008), which operates to shut down star formation in the most massive galaxies; this mechanism is also required to correctly predict the absolute and relative numbers of red galaxies at $z \lesssim 1$ (Dekel \& Birnboim 2006; Bell et al. 2007; Faber et al. 2007). With this inclusion, models thus predict that a significant fraction of massive galaxies finish their star formation relatively early in the history of the universe, with later mergers working to build up the most massive galaxies.

There is thus a crucial distinction to be made between a galaxy's mean stellar age, and the time since that galaxy has assumed its present form (see, e.g., De Lucia et al. 2006): the most massive galaxies are expected to be both the oldest and the youngest galaxies. They are the oldest in the sense that their progenitors are expected to form first in the highest cosmic overdensities. However, these stars are only assembled into their $z=0$ configuration relatively recently; in this sense, massive galaxies are expected to be rather younger than their constituent stellar populations.

This leaves (at least) two open questions related to the quenching of star formation and the formation and evolution of massive galaxies: (1) when does star formation stop in massive galaxies, and then (2) what happens to galaxies after they have stopped forming stars?

In connection with the first of these questions, deep spectroscopic surveys have identified massive galaxies with little or no ongoing star formation at $1 \lesssim z \lesssim 2$ (e.g., Cimatti 2004; Glazebrook et al. 2004; McCarthy et al. 2004; Daddi et al. 2004). At the same time, color selection techniques like the ERO (McCarthy 2004 and references therein), DRG (Franx et al. 2003), or BzK (Daddi et al. 2005) criteria have been used to identify massive, passive galaxies at moderate- to high redshifts. While these techniques are deliberately biased toward certain kinds of galaxies and certain redshift intervals, advances in techniques for photometric redshift estimation and stellar population modeling have allowed the selection of mass-limited samples, and so the construction of representative samples of the high-redshift massive galaxy population (e.g., van Dokkum et al. 2006). 
By obtaining very deep rest-frame optical spectra of a photometric redshift-selected sample of massive galaxies at $z \gtrsim 2$, Kriek et al. $(2006,2008 b)$ made a significant advance on previous spectroscopic and photometric studies. Of the $36 z_{\text {spec }}>2$, $M_{*}>10^{11} M_{\odot}$ galaxies in the Kriek et al. (2008b) sample, 16 were shown unambiguously to have evolved stellar populations and little or no ongoing star formation (see also Muzzin et al. 2009). These galaxies also seem to form a red sequence in $(B-V)$ color, although at low significance $(3.3 \sigma$; Kriek et al. 2008a; see also Kriek et al. 2009). In other words, these massive galaxies appear both to have assembled stellar populations similar to galaxies of comparable mass in the local universe, and to have had their star formation effectively quenched.

Using Keck laser guide-star-assisted adaptive optics and Hubble Space Telescope imaging, van Dokkum et al. (2008, hereafter vD08) measured sizes for 9 of the 16 strongly quenched galaxies from the Kriek et al. (2006, 2008b) sample. They found (rest-frame optical) effective radii in the range of $0.5-2.4 \mathrm{kpc}$, that is, smaller than typical galaxies of the same mass in the local universe by factors of 3-10. These galaxies have stellar mass densities, measured within the central $1 \mathrm{kpc}$, that are 2-3 times higher than typical local galaxies of the same mass (Bezanson et al. 2009). Similar sizes and densities have been found for a larger sample of 82 massive galaxies at $1.7<z<3.0$ from the GOODS survey by Buitrago et al. (2008). Cimatti et al. (2008) and Damjanov et al. (2009, hereafter D09) have found similarly compact sizes for massive galaxy samples drawn from $1<z<2$ spectroscopic surveys. Further, van Dokkum et al. (2009) have recently measured a velocity dispersion of $510_{-95}^{+165} \mathrm{~km} \mathrm{~s}^{-1}$ for one of the galaxies in the vD08 sample, based on a $29 \mathrm{hr}$ NIR spectrum; this extremely high value is consistent with the galaxy's measured mass and size. (See also Cappellari et al. 2009, who have measured velocity dispersions for two $z \sim 1.4$ galaxies and a stacked spectrum of 7 massive galaxies at $1.6<z<2.0$, and Cennaro \& Trujillo 2009, who measured a velocity dispersion for a stacked spectrum of 13 massive galaxies at $1.4<z<2.0$.) These results confirm and consolidate the work of Daddi et al. (2005), Trujillo et al. (2006, 2007), Zirm et al. (2007), and Toft et al. (2007), as well as the $1<z<2$ results from, e.g., Longhetti et al. (2007) and Saracco et al. (2009), and the $z \lesssim 1$ results from van der Wel et al. (2008).

The significance of these results is that, in terms of their stellar populations, these $z \gtrsim 2$ galaxies appear to be more or less "fully formed." Not only have they already assembled stellar populations comparable to local early-type galaxies, but they have also already had their star formation strongly quenched, to the extent that they may even form a red sequence (see also Williams et al. 2009; Brammer et al. 2009). On the other hand, in order to develop into galaxies like the ones seen in the local universe, it would seem that they must each undergo significant structural evolution. Taken together, these results thus paint a consistent picture of strong size evolution among massive, earlytype and/or red sequence galaxies ${ }^{6}$ - both individually and as a population - even after their star formation has been quenched (see also Franx et al. 2008). Whatever the mechanism for this growth in size (see, e.g., Fan et al. 2008; Hopkins et al. 2009; Naab et al. 2009; Khochfar \& Silk 2009), it would seem that the formation of massive, passive galaxies is not monolithic.

\footnotetext{
6 There is considerable, but not total, overlap between the color-selected samples of red sequence galaxies, and the morphology-selected samples of early-type galaxies. While it is common to use these terms as if they were more or less interchangeable, it should be remembered that they are not.
}

Our aim is to test the claim that there are no galaxies in the local universe with properties consistent with their being the passively evolved counterparts to the massive, passive, compact galaxies seen at $z \gtrsim 2$. In doing so, we aim to confirm (or refute) the idea that each of the galaxies seen at $z \gtrsim 2$ must undergo significant structural evolution between then and now-this is the crux of the argument against the "monolithic" formation of massive galaxies. Our search is based on many of the latest data products from the Sloan Digital Sky Survey (SDSS; York et al. 2000; Strauss et al. 2002). In particular, we will focus on the possibility that such galaxies have been overlooked in SDSS due to selection effects associated with the construction of the spectroscopic target sample.

The structure of this paper is as follows: we describe the basic SDSS data that we have used in Section 2. In Section 3, we define our sample of compact galaxy candidates, and present several checks to confirm that these galaxies are indeed unusually small for their stellar masses. Then, in Section 4, we consider the importance of the SDSS spectroscopic selection for massive, compact galaxies. In this section, we also compare our $z \sim 0.1$ compact galaxy candidates with the vD08 and D09 samples. Finally, in Section 5, we compare our results to two similar, recent studies and briefly examine the properties of our compact galaxies' stellar populations in comparison to the general $z \sim 0.1$ red sequence galaxy population.

We also provide a complementary analysis in Appendix $\mathrm{A}$, in which we search for massive, compact, red sequence galaxies in the full SDSS photometric sample, using photometric redshifts. In this way, we test our conclusion that the apparent differences between the high- and low-redshift samples cannot be explained by selection effects, and derive an estimate for the number of compact galaxies that may be missing from the SDSS spectroscopic sample.

A summary of our main results is given in Section 6 . Throughout this work, we assume the concordance cosmology (viz., $\Omega_{\mathrm{m}}=0.3, \Omega_{\Lambda}=0.7$, and $H_{0}=70 \mathrm{~km} \mathrm{~s}^{-1} \mathrm{Mpc}^{-1}$ ) and the Chabrier (2003) stellar initial mass function (IMF).

\section{BASIC DATA AND ANALYSIS}

The present work is based on Data Release 7 (DR7; Abazajian et al. 2009) of the SDSS, accessed via the Catalog Archive Server $^{7}$ (CAS; Thakar et al. 2008). In this section, we describe the different SDSS data sets that we have used and our analysis of the data. We will search for compact galaxy candidates in the SDSS spectroscopic catalog; to this end, we will only consider sciencePrimary objects (a flag indicating a "sciencegrade" spectrum, and weeding out multiple observations of individual objects) with either a star or galaxy photometric type (i.e., a genuine astronomical source). The details of the SDSS spectroscopic sample selection are given in Strauss et al. (2002); we will summarize the most relevant aspects of this process in Section 4.1.

\subsection{The Basic SDSS Catalog}

For the basic SDSS catalog, there are two different methods for performing photometry. The first, the Petrosian or petro magnitude, is derived from the observed, azimuthally averaged (one-dimensional) light profile. The Petrosian radius is defined as the point where the mean surface brightness in an annulus drops to a set fraction (viz., 0.2) of the mean surface brightness

\footnotetext{
http://casjobs.sdss.org/CasJobs/
} 
within a circular aperture of the same radius. Within SDSS, the petro aperture is defined to be twice the Petrosian radius; this aperture will contain $99 \%$ of the total light for a well-resolved exponential disk, but may miss as much as $18 \%$ of the light for a de Vaucouleurs $R^{1 / 4}$ profile (Strauss et al. 2002; Blanton et al. 2005).

The second photometric measure is derived from fits to the observed (two-dimensional) distribution of light in each band, using a sector-fitting technique, in which concentric annuli are divided into twelve $30^{\circ}$ sectors (see Appendix A.1 of Strauss et al. 2002). These fits are done assuming either an exponential or a de Vaucouleurs profile, convolved with a fit to the appropriate point-spread function (PSF). For each profile, the structural parameters (viz. axis ratio, position angle, and scale length) are determined from the $r$-band image. The more likely (in a $\chi^{2}$ sense) of the two profile fits is used to define model magnitudes for each galaxy. For the ugiz bands, these parameters are then held fixed, and only the overall normalization (i.e., total flux) is fit for.

We use model magnitudes to construct ugriz spectral energy distributions (SEDs) for each object, since these measurements are seeing-corrected. From DR7, the basic SDSS photometric calibration has been refined so that the photometry is given in the $\mathrm{AB}$ magnitude system without the need for any further corrections (Padmanabhan et al. 2008).

The basic catalog also provides two different measures of size, associated with these two magnitude measurements. The Petrosian half-light radius, $R_{50}$, is defined as the radius enclosing half the "total" petro flux. The catalog also contains bestfit structural parameters, including the effective radius, from a separate set of fits to each band independently, again for both an exponential and a de Vaucouleurs profile. Note that whereas the petro magnitude and size are derived from the observed, PSF-convolved radial profile, the model values provide a PSFcorrected measure of the intrinsic size. For measuring sizes, we will rely on the best-fit model effective radius, $R_{\mathrm{e}}$, as determined from the $z$ band.

We also adopt a minimum measured size of 0.75 , corresponding to half the median PSF FWHM for the SDSS imaging. We will plot all galaxies with (PSF-corrected) effective radii smaller than 0.75 (that is, galaxies with sizes on the order of the seeing) as upper limits. We note that this limit is rather conservative: the galaxies we are considering are typically bright enough that their growth curves are significantly different from that of a point source. (We consider the separate but related issue of star/ galaxy separation in Section 4.1 below). In any case, none of our conclusions depend on the choice of this limit, which ultimately affects only five of our lower-mass compact galaxy candidates.

\subsection{Derived Quantities}

We have derived rest-frame photometry for each object, based on its observed ugriz SED and redshift, using the IDL utility InterRest (Taylor et al. 2009), using a redshift grid of $\Delta z=0.001$. In order to minimize the $k$-corrections and their associated errors, we determine rest-frame photometry through the ugriz filters redshifted to $z=0.1$, which we denote with a superscript 0.1 (see, e.g., Blanton \& Roweis 2007). We estimate that the systematic uncertainties are at the level of $\lesssim 0.02$ mag. The agreement between our interpolated rest-frame photometry and that derived using the SDSS kcorrect algorithm (Blanton $\&$ Roweis 2007) is very good: our derived $(u-r)$ colors are $\sim 0.02$ mag bluer for blue galaxies and $\sim 0.03$ mag redder for red galaxies.
We use the stellar mass estimates derived by the MPIA Garching group. ${ }^{8}$ J.B. has fit the ugriz model photometry of all galaxies using the synthetic stellar population library described by Gallazzi et al. (2005), based on Bruzual \& Charlot (2003) models and assuming a Chabrier (2003) IMF in the range 0.1-100 $M_{\odot}$. The Gallazzi et al. (2005) library contains a large number of Monte Carlo realizations of star formation histories, parameterized by a formation time $\left(1.5<t_{\text {form }} /[\mathrm{Gyr}]<13.5\right)$, an exponential decay rate $\left(0<\gamma /\left[\mathrm{Gyr}^{-1}\right]<1\right)$, and including a number of random star formation bursts (randomly distributed between $t_{\text {form }}$ and 0 , normalized such that $10 \%$ of galaxies experience a burst in the last $2 \mathrm{Gyr}$ ). In the fitting, the photometry has been corrected for emission lines under the assumption that the global emission line contribution is the same as in the spectroscopic fiber aperture.

The agreement between these SED-fit mass estimates and those of Kauffmann et al. (2003a), which were derived from spectral line indices, are excellent: the median offset is $-0.01 \mathrm{dex}$, with a scatter on the order of $0.1 \mathrm{dex}$. For the highest masses, however, the SED-fit results are slightly less robust: for $M_{*}>10^{11} M_{\odot}$, the median formal error is $\lesssim 0.10 \mathrm{dex}$, compared to $\lesssim 0.06$ dex for the Kauffmann et al. (2003a) estimates. (Note that these uncertainties do not include, for example, uncertainties intrinsic to the stellar population models, and are thus underestimated; see Conroy et al. 2009).

In the upper panel of Figure 1, we show the stellar mass to light ratios, $M_{*} / L_{i}$, for $0.066<z<0.12$ galaxies as a function of their ${ }^{0.1}(g-i)$ color (again, $L_{i}$ should be understood as referring to the $i$-band filter redshifted to $z=0.1$, or ${ }^{0.1} i$ ). Note that, at least for these mass estimates, $M_{*} / L$ is very tightly correlated with color. In the main panel of this figure, the red line shows the median $M_{*} / L_{i}$ in narrow color bins. Making a simple linear fit to these points, we find

$$
\log \left(M_{*} / L_{i}\right)=-0.82+0.83 \times{ }^{0.1}(g-i),
$$

where both $M_{*}$ and $L_{i}$ are in solar units. (The absolute magnitude of the Sun in the ${ }^{0.1} i$ band is 4.58.) This relation is shown in Figure 1 as the solid blue line. We present this relation as an alternative to the popular Bell \& de Jong (2001) or Bell et al. (2003) relations.

In the lower panel of Figure 1, we show the dispersion around the median relation; in this figure, the error bars show the $16 / 84$ percentiles in the $M * / L$ distribution in narrow bins of color. Overall, the dispersion around this relation is just 0.032 dex. Note that while the simple linear relation given above provides an acceptable description of the "true" relation, systematic offsets exist at the 0.02-0.04 dex level. The global mean and random offset from this linear relation are $0.002 \mathrm{dex}$ and 0.040 dex, respectively.

In both panels, the small gray pluses show points that fall outside the plotted range. Note that a small fraction of galaxies lie well off the main $\left(M_{*} / L\right)$-color relation, some by an order of magnitude or more. These galaxies also lie significantly off the main stellar mass-dynamical mass relation and are very likely to represent catastrophic failures of the stellar mass SED-fitting algorithm (see Section 3.1 below). This presents a problem when it comes to looking for outliers in the mass-size plot: selecting the most extreme objects may well include those objects with the largest errors. For this reason, we will restrict our attention to those objects that fall within $0.25 \mathrm{dex}(\approx 7.8 \sigma)$ of the main

\footnotetext{
8 Available via http://www.mpa-garching.mpg.de/SDSS/DR7/Data/ stellarmass.html.
} 


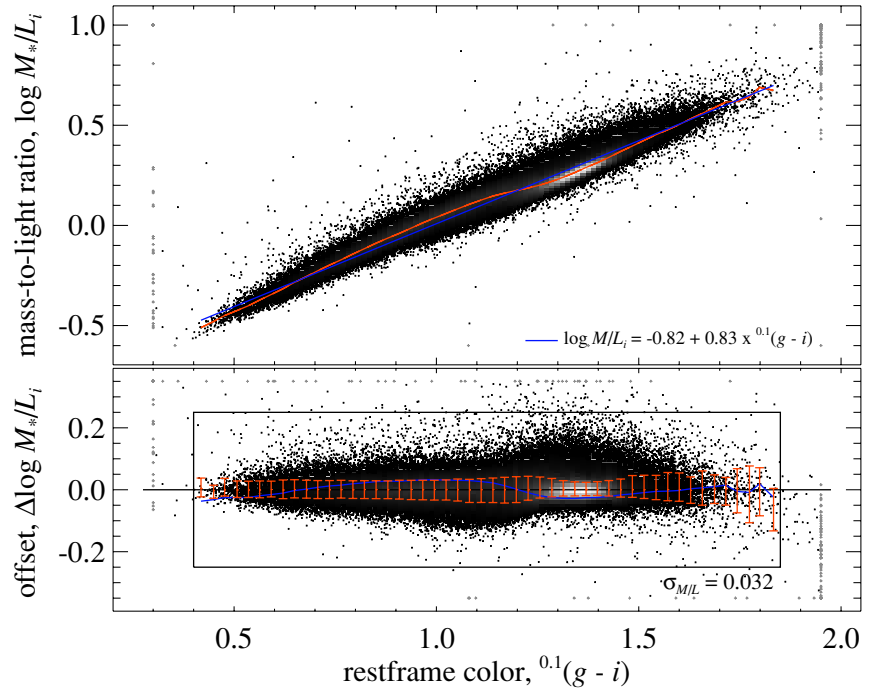

Figure 1. Mass-to-light ratios, $M_{*} / L_{i}$, of $0.066<z<0.12$ galaxies as a function of their ${ }^{0.1}(g-i)$ color. The gray scale shows the (linear) data density in cells, where the data density is high. In the main panel, the red line shows the median $M_{*} / L_{i}$ in narrow bins of ${ }^{0.1}(g-i)$ color; the blue line is a linear fit to these points. (Here, $M_{*} / L_{i}$ should be understood to relate to the $i$ band redshifted to $z=0.1$.) In the lower panel, we have simply subtracted away the median relation; in this panel, the error bars show the $16 / 84$ percentiles in color bins. The simple linear relation shown provides an acceptable description of the observed relation, to within 0.02-0.04 dex; the global rms offset from this relation is 0.032 dex. In order to avoid selecting "catastrophic failures" in terms of stellar mass estimates, we will consider only those galaxies that have $0.4<{ }^{0.1}(g-i)<1.8$, and that fall within 0.25 dex of the median $M_{*} / L_{i}-^{0.1}(g-i)$ relation; this selection is shown by the box in the lower panel. (A color version of this figure is available in the online journal.)

$\left(M_{*} / L\right)$-color relation, and with $0.2<{ }^{0.1}(g-i)<1.8$, as shown by the box in the lower panel of Figure 1 . This selection excludes just under 600 of the 223,292 galaxies shown in Figure 1.

\section{SEARCHING FOR MASSIVE, COMPACT, EARLY-TYPE GALAXIES IN THE LOCAL UNIVERSE}

\subsection{Identifying Massive, Compact Galaxy Candidates}

Figure 2 shows the size-mass plot for a sample of massive, red sequence galaxies drawn from the SDSS DR7 spectroscopic catalog; this sample has been selected to have ${ }^{0.1}(u-r)>2.5$ in the range $0.066<z<0.12$. Since we are interested primarily in potential passively evolved analogs to the $z \gtrsim 1.5$ galaxies seen by vD08 and D09, most of our analysis will focus on this red sequence sample; we will briefly consider massive, compact, blue galaxies in Section 5.2.

We have chosen our redshift limits to minimize the importance of selection effects and measurement biases, which we will discuss in Section 4.1. For now, we note that, mapping the $m_{r}<17.77$ spectroscopic limit onto $M_{*}(z)$, we should be highly complete (volume limited) for $M_{*}>10^{10.7} M_{\odot}$ and $z<0.12$. As a very simple check on this, we note that for this sample, the median redshift in narrow bins of stellar mass is within the range $z=0.098-0.102$ for all $M_{*}>10^{10.7} M_{\odot}$; the volumetric center of the $0.066<z<0.12$ bin is $z=0.10$.

The yellow points in this figure show the median size in narrow bins of stellar mass; the error bars show the 14/86 percentiles. For comparison, the long-dashed line shows the local size-mass relation for early-type galaxies from Shen et al. (2003), corrected for differences in assumed IMF and cosmology. Contrary to the findings of Valentinuzzi et al. (2010), a simple fit to the size-mass relation for red sequence galaxies $\left({ }^{0.1}(u-r)>2.5\right)$ shown in Figure 2 is consistent with the Shen et al. (2003) relation for early-type $(n>2.5)$ galaxies, albeit offset by -0.05 dex in size, or, equivalently, by -0.09 dex in mass. At fixed mass, the mode of the distribution is similarly offset (see Figure 7); this does not appear to be due to large numbers of late-type galaxies in our sample of red sequence galaxies.

We next select and study very compact galaxies from within the red sequence sample shown in Figure 2. At first glance, it appears that there may be a few galaxies that lie well below the main size-mass relation. However, it must be remembered that by selecting the most extreme outliers, we will also be selecting those objects with most egregious measurement errors.

For this reason, we have individually visually inspected all $M_{*}>10^{10.7} M_{\odot}$ galaxies with inferred sizes that are less than half the size predicted from the Shen et al. (2003) relation; i.e.,

$$
\log \left(R_{\mathrm{e}} / \mathrm{kpc}\right)<0.56 \times\left(\log \left(M_{*} / M_{\odot}\right)-9.84\right)-0.3 .
$$

For sizes smaller than the median relation, the distribution of sizes around the Shen et al. (2003) relation is very well described by a log-normal with $\sigma=0.11 \mathrm{dex}$; this $\Delta R_{\mathrm{e}}$ cut thus corresponds to selecting those galaxies whose sizes are smaller than the mean size (at fixed mass) at the $\gtrsim 2.7 \sigma$ level. (Adopting our own fit to the size-mass relation, this selection translates to $\Delta R_{\mathrm{e}}<-0.35$ dex; our results are otherwise unchanged.)

We have inspected 280 such objects, and discarded those where there are obvious reasons to distrust the size measurements. The most common reasons for discarding galaxies are confusion with other galaxies (99 galaxies, including 19 good merger candidates, and 2 possible lenses), or with the extended halos, diffraction spikes, and/or reflections of bright stars (62 galaxies). Another 19 galaxies were clearly disk-like, 5 showed marked asymmetries, and 1 had a very strong active galactic nucleus (AGN) spectrum; these candidates were also discarded. We discarded a further three objects with bad or missing data.

In Figure 3, we show several illustrative examples of the galaxies we are considering. On the right-hand side of this figure, we show a "normal" early-type galaxy, with $M_{*} \approx 10^{11} M_{\odot}$, which falls very close to the Shen et al. (2003) relation. Below this, we show two of the compact galaxy candidates that we have rejected on the basis of visual inspection. On the lefthand side of this figure, we show three of the compact galaxy candidates of different stellar masses that we have retained after visual inspection. For each galaxy, we show the thumbnail image from the SDSS SkyServer, ${ }^{9}$ used for visual inspection. We also show each galaxy's observed spectrum and photometry; here, we have scaled the photometry to match to the integrated $r$-band flux from the observed spectrum.

In addition to these galaxies with suspect size measurements, we have excluded 27 galaxies whose SED-fit $M_{*} / L$ s are offset from the main color $-\left(M_{*} / L\right)$ relation shown in Figure 1 by more than 0.25 dex. If we use Equation (1) to derive new stellar mass estimates for these galaxies, all of these galaxies move back into the main cloud in both Figure 2 and a stellar massdynamical mass plot, with mean/median offsets of $\lesssim 0.02$ dex in both cases. This strongly suggests that the SED-fit $M_{*} / L$ s for these 27 galaxies are simply wrong.

The 190 galaxies discarded on the basis of inspection are shown in Figure 2 as small red crosses; the small blue crosses

\footnotetext{
9 Also accessible via CAS at http://cas.sdss.org.
} 


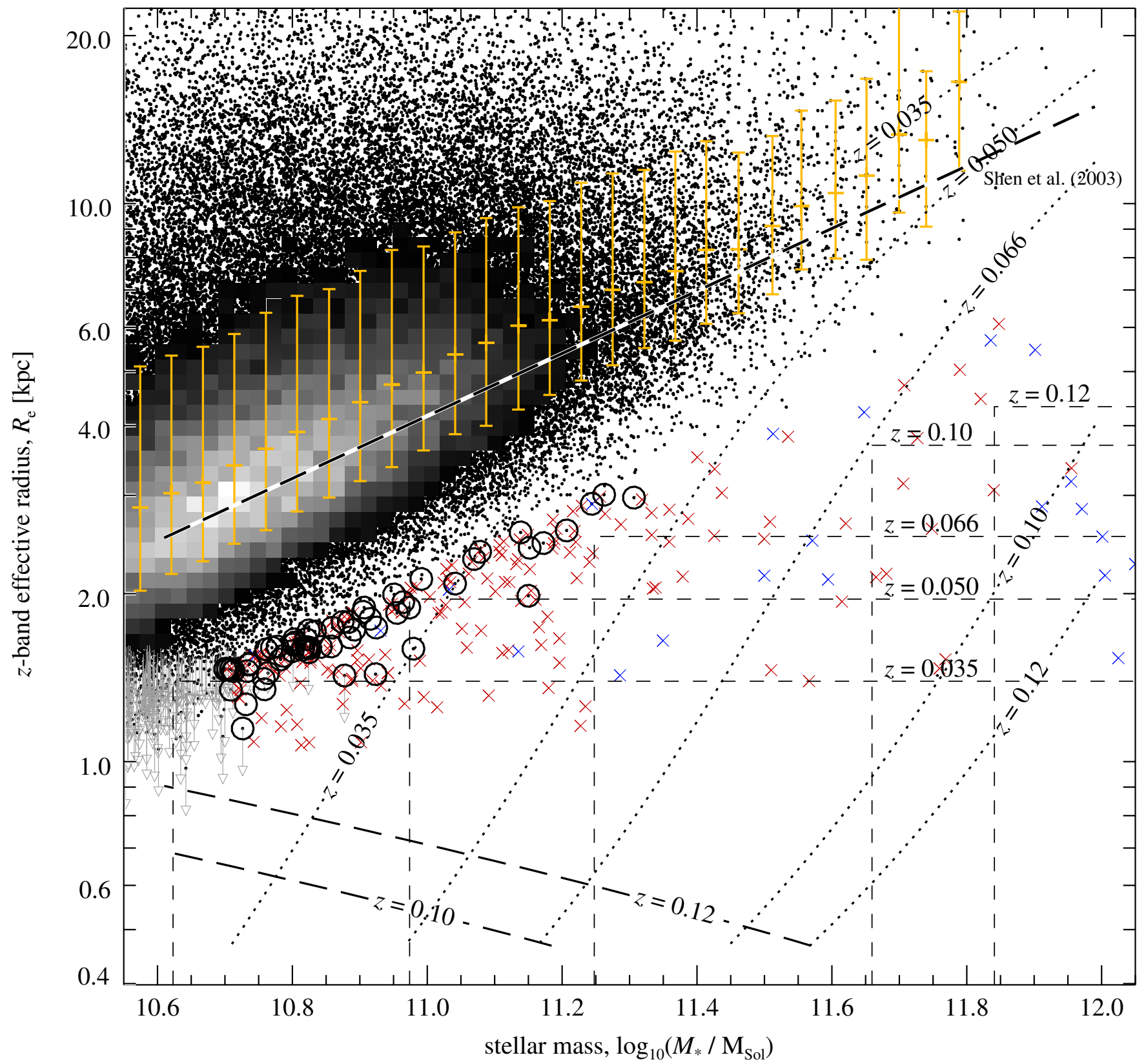

Figure 2. Size-mass relation for massive, red sequence galaxies, illustrating the importance of the SDSS spectroscopic selection criteria. The points show SDSS galaxies $(0.066<z<0.12)$ selected to have ${ }^{0.1}(u-r)>2.5$. The yellow points show the median size in narrow bins of stellar mass; the error bars show the $16 / 84$ percentiles in each bin. A fit to this median size-mass relation for red sequence galaxies is consistent with the Shen et al. (2003) relation for early-type galaxies (dashed line), albeit offset by $0.05 \mathrm{dex}$ in $R_{\mathrm{e}}$. Individual galaxies that we have visually inspected $\left(M_{*}>10^{10.7} M_{\odot} ; \Delta \log R_{\mathrm{e}}<-0.3\right.$ dex) are marked with large symbols. Galaxies with $M / L \mathrm{~s}$ that differ significantly from the main color- $(M / L)$ relation shown in Figure 1 are marked with small blue crosses. Galaxies with obvious problems in their photometry (especially those affected by the presence of a bright nearby star or blended with other galaxies) are marked with a small red cross; those that look okay are plotted as circles. Galaxies with observed sizes smaller than 0 '.75 are plotted as upper limits, assuming a size of 0.75 . The different lines show how the principal selection limits for spectroscopic follow-up translate onto the $\left(M_{*}, R_{\mathrm{e}}\right)$ plane for $z=0.12,0.10,0.066,0.050$, and 0.35 (top to bottom): the diagonal, long-dashed lines show the star/galaxy discriminator; the short-dashed boxes show the "saturation" selection limit, and the diagonal dotted lines show the "cross-talk" selection limit (see Section 4.1 for a detailed discussion). Galaxies lying below these lines will not be targeted spectroscopically. Note that, at least for massive galaxies, the star/galaxy separation criterion is not a major source of incompleteness for $z \leqslant 0.10$. Because both the "cross-talk" and "saturation" criteria exclude high surface brightness objects, the SDSS spectroscopic sample is potentially highly incomplete for bright, compact galaxies for low redshifts. For instance, the "cross-talk" selection criterion cuts out a large fraction of the massive galaxy population, including the majority of $M_{*} \gtrsim 10^{11.5} M_{\odot}$ red sequence galaxies, at $z \lesssim 0.35$. In the $0.066<z<0.12$ interval that we consider, the "saturation" criterion is the biggest potential cause of incompleteness.

(A color version of this figure is available in the online journal.)

show the 27 galaxies with discrepant $M_{*} / L \mathrm{~s}$. As a function of $\Delta R_{\mathrm{e}}$, the fraction of inspected sources that have been discarded goes fairly smoothly from $60 \%$ for $\Delta R_{\mathrm{e}} \sim-0.3$ dex to $\sim 100 \%$ for $\Delta R_{\mathrm{e}}<-0.5$ dex. The discarded fraction has a similar dependence on mass: it is $\sim 60 \%$ for $M_{*} \sim 10^{10.7} M_{\odot}$, rising to $\sim 85 \%$ for $M_{*} \sim 10^{11} M_{\odot}$, and $100 \%$ for $M_{*}>10^{11.4} M_{\odot}$.
This leaves us with a sample of 63 massive, compact, earlytype, and red sequence galaxy candidates; these are marked in Figure 2 with heavy black circles. Of those galaxies that we have retained, $8 \%(5 / 63)$ have observed sizes smaller than 0.75 ; all of these have $M_{*}<10^{11} M_{\odot}$. We have provided the properties of our compact galaxy candidates in Table 1. 

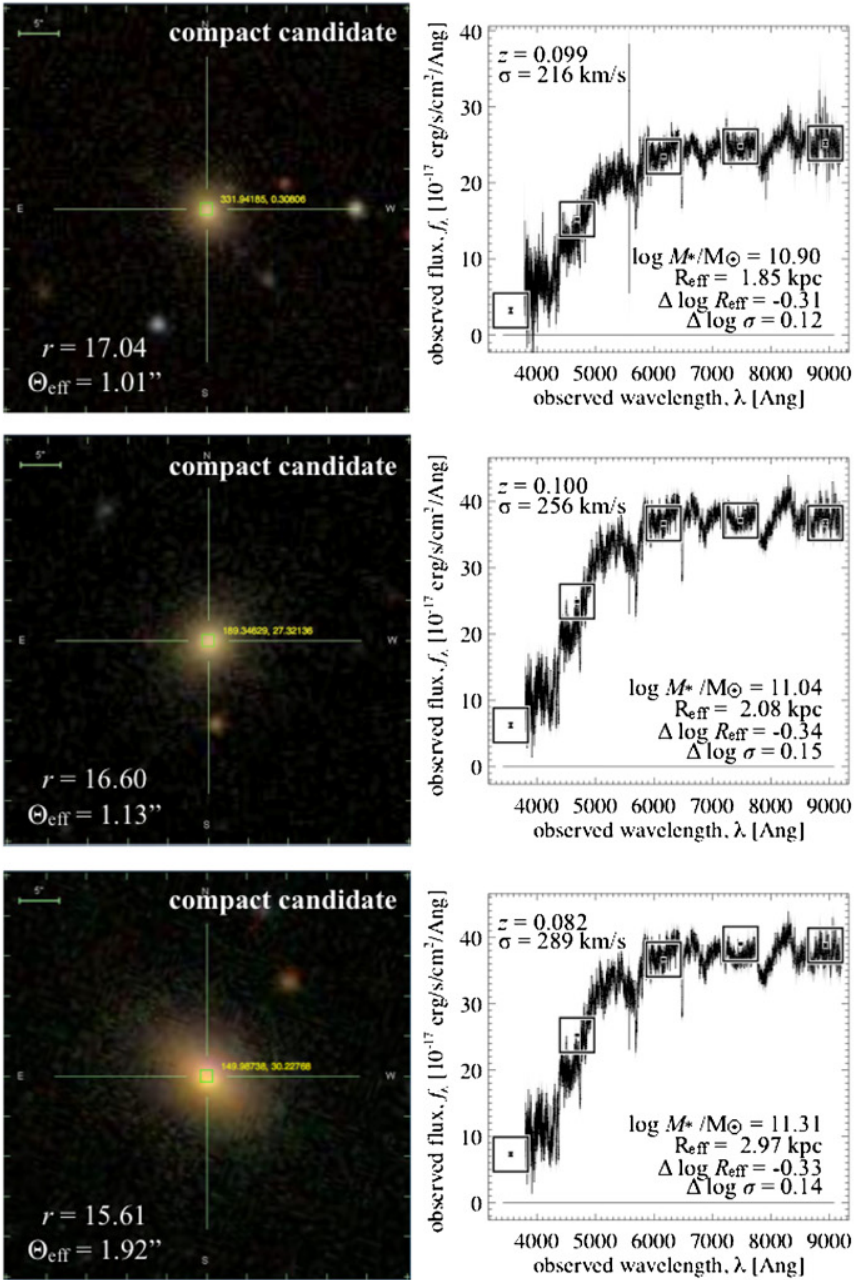
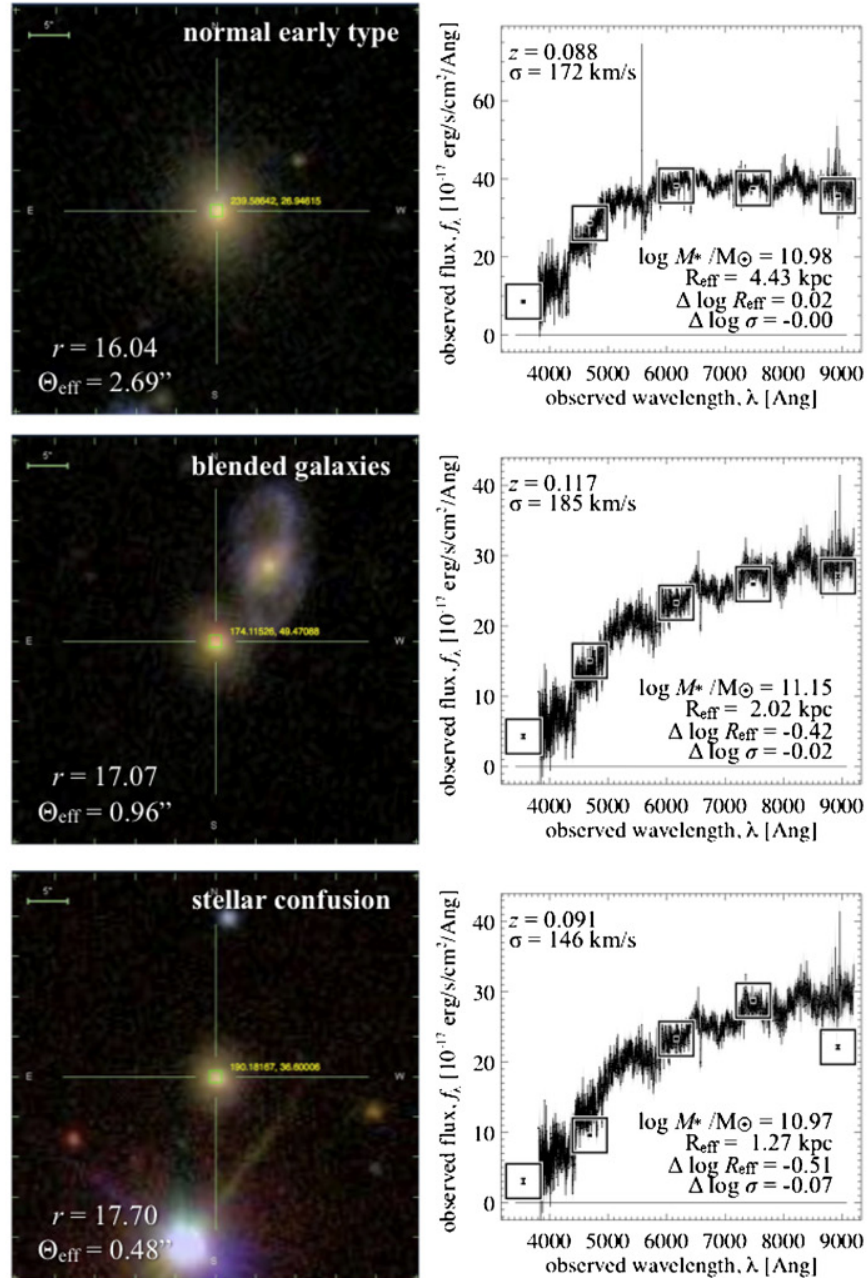

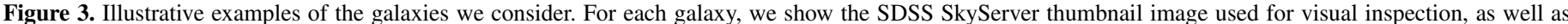

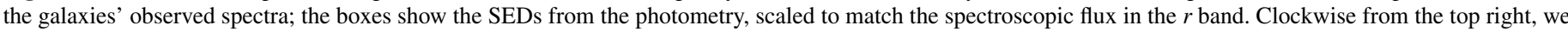

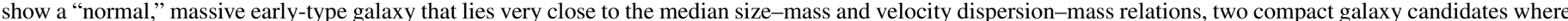

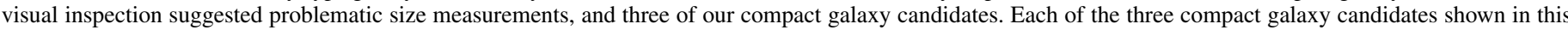
figure has observed velocity dispersions that are approximately consistent with their small measured sizes (see Section 3.3).

(A color version of this figure is available in the online journal.)

\subsection{Are the Size Measurements Wrong?}

We have performed a number of checks to validate the small measured sizes of our compact galaxy candidates. The compact galaxy candidates do not have significantly larger size measurement errors in comparison to the full sample shown in Figure 2. For both the $r$ and $z$ bands, our candidates are not anomalous in a plot of petro half-light radius versus model effective radius, nor are they anomalous in a plot of $r$-band size versus $z$-band size. For all but two of the candidates, the petro and model magnitudes agree to within 0.15 mag. The mean offset between model and petro magnitudes is -0.06 mag for the compact galaxies, compared to -0.08 mag for the full sample shown in Figure 2. That is, the compact candidates appear to be well described by the de Vaucouleurs model fits.

For the New York University (NYU) Value Added Galaxy Catalog (VAGC), Blanton et al. (2005) have made Sérsic-profile fits to the radially averaged light profiles of each object, in which they allowed the free parameter $n$ to vary over the range $0 \leqslant n<6$. In order to explore further the issue of the quality of the de Vaucouleurs model fits, we have compared the model effective radii to those given in the VAGC.
In Figure 4, we show the distribution of Sérsic parameters for our candidates, as well as a comparison between the Sérsic and de Vaucouleurs sizes. First, we note that nearly all (59/63) of our compact galaxy candidates have $n>3$; these are not latetype (exponential) galaxies. It is therefore unsurprising-but nonetheless reassuring - that the two size measures agree quite well: for the median galaxy among our candidates, the de Vaucouleurs size is $\sim 10 \%$ smaller than the Sérsic size; the rms dispersion is 0.10 dex. For comparison, the median quoted error for the de Vaucouleurs size measurements is $4.6 \%$.

Note that $\approx 25 \%(17 / 63)$ of our candidates have $n=5.9$ in the NYU VAGC (the maximum value allowed in the fits). These galaxies are considerably more centrally concentrated than the canonical de Vaucouleurs $R^{1 / 4}$-law profile. However, the trend with increasing Sérsic index is for the de Vaucouleurs size, $R_{\mathrm{DeV}}$, to be systematically lower than the Sérsic size, $R_{\mathrm{Ser}}$ : making a least-squares fit to the data shown in Figure 4 , we find $\log R_{\mathrm{DeV}} / R_{\mathrm{Ser}}=-0.02-0.05(n-4)$. If these galaxies do have $n>6$, then we may well be underestimating their sizes by $\gtrsim 25 \%$.

Guo et al. (2009) have recently demonstrated that as a result of biases in the way the background sky level is estimated for 
Table 1

Properties of our Compact Galaxy Candidates

\begin{tabular}{|c|c|c|c|c|c|c|c|c|c|c|c|c|}
\hline $\begin{array}{l}\text { R.A. } \\
(1)^{\mathrm{a}}\end{array}$ & $\begin{array}{l}\text { Decl. } \\
(2)^{\mathrm{a}}\end{array}$ & $\begin{array}{c}z \\
(3)^{\mathrm{a}} \\
\end{array}$ & $\begin{array}{c}(u-r)_{\mathrm{obs}} \\
(4)^{\mathrm{a}}\end{array}$ & $\begin{array}{c}0.1(u-r) \\
(5)\end{array}$ & $\begin{array}{c}\log M_{*} \\
(6)^{\mathrm{b}} \\
\end{array}$ & $\begin{array}{l}\Theta_{50, z} \\
(7)^{\mathrm{a}}\end{array}$ & $\begin{array}{c}n \\
(8)^{\mathrm{c}} \\
\end{array}$ & $\begin{array}{c}\Theta_{50, z} \\
(9)^{\mathrm{c}}\end{array}$ & $\begin{array}{c}R_{\mathrm{e}} \\
(10)\end{array}$ & $\begin{array}{c}\sigma \\
(11)^{\mathrm{b}} \\
\end{array}$ & $\begin{array}{c}T \\
(12)^{\mathrm{d}}\end{array}$ & $\begin{array}{c}Z \\
(13)^{\mathrm{d}} \\
\end{array}$ \\
\hline 190.16652 & 13.81563 & 0.0855 & 2.577 & 2.642 & 10.700 & 0.912 & 4.31 & 0.917 & 1.462 & 160 & $\ldots$ & $\ldots$ \\
\hline 127.02722 & 55.37988 & 0.0665 & 2.828 & 2.999 & 10.701 & 1.152 & 3.26 & 1.211 & 1.468 & 191 & $\ldots$ & $\ldots$ \\
\hline 225.31708 & 30.58266 & 0.0980 & 2.858 & 2.877 & 10.705 & 0.808 & 3.87 & 0.773 & 1.464 & 195 & $\ldots$ & $\ldots$ \\
\hline 227.08531 & 7.25325 & 0.0764 & 2.963 & 3.113 & 10.709 & 0.929 & 5.13 & 1.188 & 1.345 & 199 & $\ldots$ & $\ldots$ \\
\hline 215.41043 & 40.03233 & 0.0998 & 2.803 & 2.813 & 10.709 & 0.795 & 4.11 & 0.670 & 1.464 & 176 & 9.775 & 0.035 \\
\hline 222.12988 & 26.48791 & 0.1058 & 2.549 & 2.540 & 10.712 & 0.750 & 4.65 & 0.722 & 1.453 & 155 & $\ldots$ & $\ldots$ \\
\hline 118.81702 & 33.22864 & 0.0980 & 2.680 & 2.697 & 10.713 & 0.803 & 2.67 & 0.739 & 1.454 & 154 & -99 & -99 \\
\hline 143.05707 & 11.70454 & 0.0811 & 2.690 & 2.776 & 10.726 & 0.750 & 5.52 & 0.830 & 1.146 & 166 & 9.255 & 0.132 \\
\hline 204.66577 & 59.81854 & 0.0704 & 2.857 & 3.012 & 10.731 & 0.943 & 3.92 & 0.974 & 1.267 & 235 & 9.845 & 0.229 \\
\hline 230.28553 & 24.21978 & 0.0809 & 2.922 & 3.028 & 10.733 & 0.952 & 5.90 & 1.298 & 1.453 & 153 & $\ldots$ & $\ldots$ \\
\hline
\end{tabular}

Notes. Properties of our compact galaxy candidates. Columns 1 and 2: position in decimal degrees (J2000); Column 3: spectroscopic redshift; Columns 4 and 5: observed and rest-frame colors; Column 6: stellar mass in units of solar masses; Column 7: apparent de Vaucouleurs model effective radius in arcsec; Columns 8 and 9: Sérsic index and apparent Sérsic effective radius in arcsec; Column 10: physical de Vaucouleurs effective radius in kpc; Column 11: velocity dispersion in $\mathrm{km} \mathrm{s}^{-1}$; Column 12: luminosity-weighted age in Gyr; Column 13: mean metallicity. In Columns 12 and 13, data are only given for those objects that appear in DR4; values of -99 indicate unsuccessful fits to the spectra. Note that we give only the first 10 candidates here; the properties of the full sample of 63 galaxies is available as an electronic table via http://www.strw.leidenuniv.nl/ ent/.

${ }^{a}$ The default SDSS (York et al. 2000; Strauss et al. 2002) catalog for DR7 (Abazajian et al. 2009), accessed via CAS (Thakar et al. 2008).

b The MPA-JHU catalog for DR7 (accessible via http://www.mpa-garching.mpg.de/SDSS/DR7/).

${ }^{c}$ The NYU VAGC (Blanton et al. 2005) for DR7.

d The stellar age and metallicity catalog of Gallazzi et al. (2005) for DR4.

(This table is available in its entirety in a machine-readable form in the online journal. A portion is shown here for guidance regarding its form and content.)
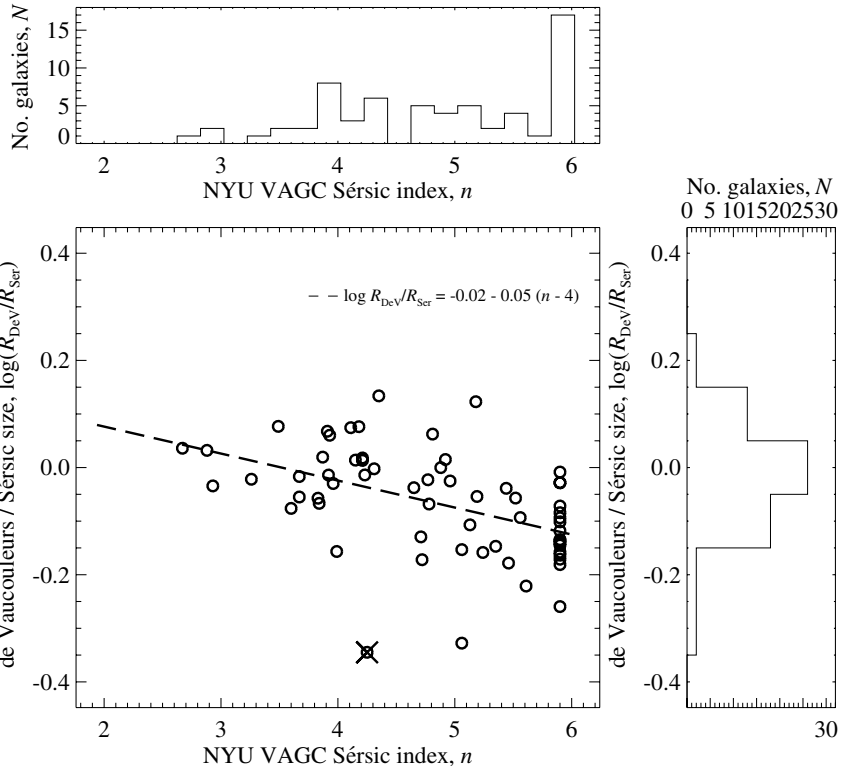

Figure 4. Comparison between effective radii derived assuming a de Vaucouleurs $(n=4)$ profile and assuming a Sérsic profile $(0<n<6)$. Whereas the basic SDSS catalog uses a sector-fitting technique to fit either an exponential $(n=1)$ or a de Vaucouleurs $(n=4)$ profile, for the NYU VAGC, Blanton et al. (2005) have fit the radial profiles of each object assuming a general Sérsic model $(0<n<6)$. This figure shows the ratio of these two sizes for our compact galaxy candidates, based on the $z$-band data, as a function of Sérsic index $n$. Almost all candidates have $n>3$; these galaxies are not exponentials. However, approximately $25 \%$ have $n=5.9$; the maximum value allowed in the Blanton et al. (2005) fits. For these galaxies, the median ratio between the two size measurements is 0.88 , with an rms scatter of 0.1 dex. In the main panel, we show a least-squares fit to the data; the dispersion around this relation is $\lesssim 0.1$ dex.

the Sérsic fits, the NYU-VAGC sizes are systematically underestimated at the $\gtrsim 15 \%$ level for $n \gtrsim 5$. This problem becomes progressively worse for large sizes $\left(\Theta_{\mathrm{e}} \gtrsim 1^{\prime \prime}\right)$ and bright magnitudes $\left(m_{r} \lesssim 16\right)$; for our compact galaxy candidates, the effect is likely to be at the $\sim 20 \%$ level. But note this implies that the difference between the de Vaucoleurs and Sérsic sizes is even greater than Figure 4 might suggest: the sizes of the $n \gtrsim 5$ compact galaxies may be underestimated by as much as $\gtrsim 30 \%$.

As a final check, therefore, we have also rederived Sérsic effective radii for our compact galaxy candidates using GALFIT (Peng et al. 2002) and done a similar comparison as for the NYU VAGC sizes. The agreement between the GALFIT and VAGC Sérsic indices is quite good, with an rms difference in $n$ of 1.1. Again the vast majority of objects have $n>3$. There are 19 objects that are assigned the maximum allowed value of $n=8$, but only 9 of these have $n=5.9$ in the VAGC. Making a similar fit to the difference between the de Vaucouleurs model and the GALFIT Sérsic effective radii, we find $\log R_{\mathrm{DeV}} / R_{\mathrm{Ser}}=0.08-0.08(n-4)$. As before, we may be underestimating the sizes of high- $n$ galaxies by $10 \%-35 \%$; at the same time this comparison does also suggest that we may well be overestimating the sizes of the few candidates with $n<4$. The median galaxy has a GALFIT Sérsic effective radius $15 \%$ smaller than the default de Vaucouleurs value. Lastly, we note that there is a definite mass dependence of the agreement between the GALFIT Sérsic and default de Vaucouleurs effective radii, such that all but one of the galaxies for which the sizes agree to within $20 \%$ have $M_{*}>10^{11} M_{\odot}$.

To summarize the results of this section, comparisons to onedimensional and two-dimensional Sérsic fits do not suggest that the de Vaucouleurs model effective radii are catastrophically wrong for our compact galaxy candidates. If anything, we may in fact be underestimating the sizes of these galaxies by $10 \%-30 \%$. Note that using underestimates of the sizes of local galaxies will effectively reduce the size of any apparent discrepancy in comparison to the compact, high-redshift galaxies.

\subsection{A Consistency Check Based on Velocity Dispersions}

Assuming that elliptical galaxies are structurally self-similar, the virial theorem implies that $M_{*} \propto R_{\mathrm{e}} \sigma^{2}$. At fixed mass, 

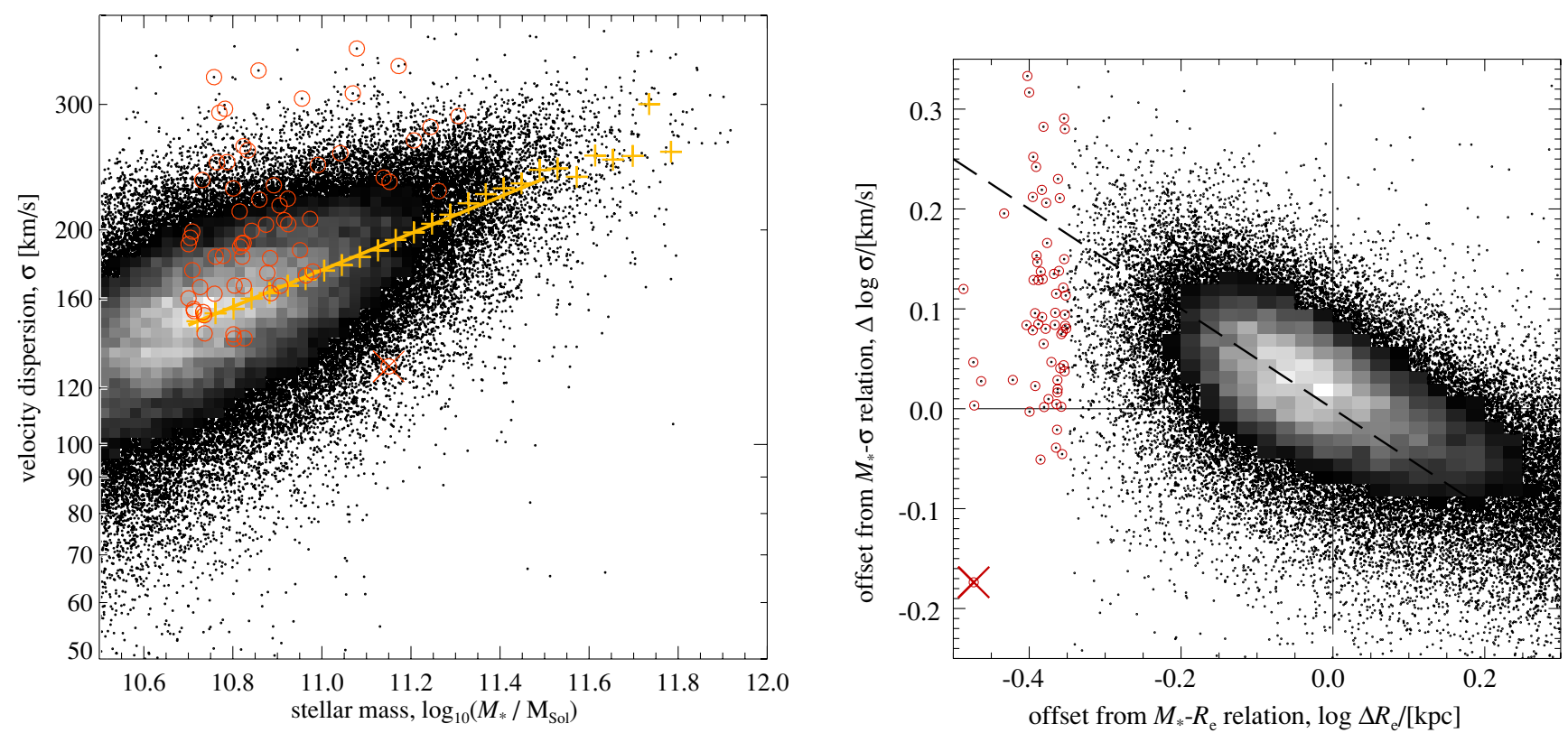

Figure 5. Using observed velocity dispersions to validate the measured sizes of our compact galaxy candidates. Left panel: the mass-velocity dispersion relation for red sequence galaxies at $0.066<z<0.12$. The points and gray scale show the SDSS data. The yellow plusses show the median velocity dispersion in narrow bins of stellar mass; the solid yellow line shows a simple fit to these points for $10.7<\log M_{*}<11.5$. The red circles highlight our compact galaxy candidates. Right panel: the offset from the $M_{*}-R_{\mathrm{e}}$ relation, plotted against the offset from the $M_{*}-\sigma$ relation for $M_{*}>10^{10.7} M_{\odot}$ galaxies with ${ }^{0.1}(u-r)>2.5$. If the offsets from these two relations are a function of galaxy size, then we expect $\Delta \log \sigma=-0.5 \times \Delta \log R_{\mathrm{e}}$ (long dashed line). Our compact galaxy candidates are shown as the red circles. In general, the observed velocity dispersions support the idea that our compact galaxy candidates are indeed relatively small; this is particularly true for those with $\sigma>200 \mathrm{~km} \mathrm{~s}^{-1}$. There is one clear exception, marked with a cross in both panels; this galaxy is also the most extreme outlier in Figure 4.

(A color version of this figure is available in the online journal.)

galaxies with small sizes should therefore have higher velocity dispersions, with $\Delta \log \sigma \approx-0.5 \times \Delta \log R_{\mathrm{e}}$, where $\Delta \log \sigma$ and $\Delta \log R_{\mathrm{e}}$ are the offsets from the $M_{*}-\sigma$ and size-mass relations for local early-type galaxies, respectively.

To determine whether the observed velocity dispersions of our compact galaxy candidates are consistent with their inferred sizes and masses, in the right-hand panel of Figure 5 we plot $\Delta \log R_{\mathrm{e}}$ against $\Delta \log \sigma$. The $M_{*}-\sigma$ relation itself is shown in the left-hand panel of the figure. (Note that for this plot, we have shifted the Shen et al. (2003) relation upward in size by 0.05 dex to be consistent with the present data set; our conclusions do not depend on this decision.) The gray scale and points show those $0.066<z<0.12$ galaxies with ${ }^{0.1}(u-r)>2.5$ and $M_{*}>10^{10.7} M_{\odot}$; the red circles indicate our compact galaxy candidates.

For the galaxies that we have identified as "compact," the median offset from the size-mass relation is $\Delta \log R_{\mathrm{e}}=-0.38$ dex. We would therefore expect a median offset from the $M_{*}-\sigma$ relation of $\Delta \log \sigma=-0.5 \times-0.38=0.19 \mathrm{dex}$. The median value for $\Delta \log \sigma$ is 0.12 dex-roughly $85 \%$ of the expected value, and $\sim 1.5$ times greater than the intrinsic scatter in the relation. Overall, these results are fairly consistent, although they do indicate that the sizes may be underestimated and/or the masses may be overestimated at the level of $10 \%-20 \%$. We note that the difference between the default SDSS and the NYU VAGC size measurements can account for at least half of this effect (see Section 3.2).

There is one of our compact galaxy candidates, marked in Figure 5 with a cross, whose velocity dispersion is clearly inconsistent with its being massive and compact; indeed, it has the lowest observed velocity dispersions of all of our compact galaxy candidates. This galaxy is also the biggest outlier in Figure 4. We will discuss this object in more detail in Section 4.2.
We also note that the observed velocity dispersions of the most extreme outliers from the size-mass relation $\left(\Delta \log R_{\mathrm{e}} \lesssim-0.4\right)$ are only marginally higher than for galaxies with "normal" sizes. Only one of these candidates $\left(\log M_{*}=10.73\right)$ has $\Delta \log \sigma>0.18$ dex; the median value of $\Delta \log \sigma$ is 0.03 dex. It would seem that the effects of "outlier noise" (i.e., objects being pushed to the edge of the observed distribution by measurement errors, rather than by virtue of their true, intrinsic properties) become dominant at these very extreme values of $\Delta \log R_{\mathrm{e}}$.

With these caveats, the observed velocity dispersions generally support the idea that the offsets from both the $M_{*}-R_{\mathrm{e}}$ and $M_{*}-\sigma$ relations for our compact galaxy candidates can be explained by their having small sizes for their masses/velocity dispersions.

\section{THE IMPORTANCE OF SELECTION EFFECTS FOR MASSIVE, COMPACT GALAXIES}

\subsection{SDSS Spectroscopic Sample Selection}

In order to be targeted for SDSS spectroscopic follow-up (and thus to appear in Figure 2), galaxies have to satisfy a complicated set of selection criteria (Strauss et al. 2002). In brief, there is a magnitude cut: objects must be detected at $>5 \sigma$ significance in the $r$ band, and have $m_{\mathrm{Pet}, r}<17.77$. Any objects that have been marked as blended and then segmented into smaller objects are rejected, as are any objects that include saturated pixels, or have been deblended from objects with saturated pixels. There are also a series of (low) surface-brightness-dependent criteria that are not relevant here. There are three further selection criteria that are particularly important for the relatively bright, compact galaxies we are interested in here.

The first of these is the star/galaxy separation criterion. Star/galaxy separation is done on the basis of the difference 
between the PSF and model magnitudes in the $r$ band. Here, the PSF magnitude, $m_{\mathrm{PSF}}$, is derived by fitting the surface brightness profile of each object with the appropriate PSF model. Analogous to the exponential/de Vaucouleurs model fits described in Section 2.1, the profile shape is kept fixed in the fitting, so that only the overall normalization is allowed to vary. The value of the $m_{\mathrm{PSF}}$ is then defined as the flux implied by this fit within a 7".4 aperture. Specifically, objects are only selected for spectroscopic follow-up where

$$
\Delta_{\mathrm{SG}} \equiv m_{\mathrm{PSF}, r}-m_{\mathrm{mod}, r} \geqslant 0.3 \mathrm{mag} .
$$

Here, $m_{\bmod , r}$ is the model $r$-band magnitude, as described in Section 2.1. The star/galaxy discriminator thus selects against unresolved objects.

Further to this selection, there are two separate selections that operate against high surface brightness objects. The first is designed to avoid cross-talk between spectroscopic fibers: any objects with fiber magnitudes $m_{\mathrm{fib}, g}<15, m_{\mathrm{fib}, r}<15$, and $m_{\text {fib }, i}<14.5$ are rejected. Finally, all objects with $m_{\text {Pet,r }}<15$ and a Petrosian radius $\Theta_{\text {Pet }}<2^{\prime \prime}$ are rejected. This last criterion was introduced to eliminate "a small number of bright stars that managed to satisfy [Equation (3)] during the commissioning phase of the survey, when the star/galaxy separation threshold was $\Delta_{\mathrm{SG}}=0.15 \mathrm{mag}$, and was retained for later runs to avoid saturating the spectroscopic CCDs" (Strauss et al. 2002). Strauss et al. (2002) also note that of the approximately 240,000 $m_{\text {Pet }, r}<17.77$ objects in runs 752 and 756 , none were rejected by the $m_{\mathrm{Pet}, r}<15, \Theta_{\mathrm{Pet}}<2^{\prime \prime}$ criterion alone.

In order to model these selections, we need to relate the relevant observed quantities (viz., the apparent petro magnitude, $m_{\mathrm{Pet}, r}$, gri fiber magnitudes, the apparent Petrosian size, $\Theta_{\mathrm{Pet}}$, and the star/galaxy separation parameter, $\Delta_{\mathrm{SG}}$ ) to intrinsic size and stellar mass.

For a given redshift/distance, the intrinsic size can be trivially related to the observed effective radius, $\Theta_{\mathrm{e}}$. In order to relate $m_{\text {Pet } r}$ to $M_{*}$, we have made a simple fit to the relation between stellar mass and absolute magnitude in the observer's frame $r$ band (i.e., with no $k$-correction) for red sequence galaxies at $0.066<z<0.12$ with $M_{r}>-21$. Note that this method naturally accounts for mass-dependent trends in, e.g., metallicity along the red sequence. The scatter around this relation is $\sim 0.06 \mathrm{dex}$, with no obvious magnitude dependence. We have derived similar relations for both $m_{\mathrm{Pet}, g}$ and $m_{\mathrm{Pet}, i}$. We have derived similar empirical relations for $\Theta_{\mathrm{Pet}}, \Delta_{\mathrm{SG}}$, and the difference between the petro and fiber magnitudes, $\Delta_{\mathrm{fib}}=m_{\mathrm{Pet}}-m_{\mathrm{fib}}$, as functions of $\Theta_{\mathrm{e}}$ and $m_{\mathrm{Pet}, r}$, using the sample of massive, red sequence galaxies shown in Figure 2. The scatter around these relations is $0.059 \mathrm{dex}(15 \%), 0.18$ mag (18\%), and 0.11 mag (9\%) respectively, with no obvious systematic residuals.

Note that there is a danger of circularity in this argument: any objects that do not satisfy the selection criteria will not be present in the sample that we use to model the selection criteria. The crucial assumption here, then, is that we can extrapolate the functions for $\Theta_{\mathrm{Pet}}\left(\Theta_{\mathrm{e}}, m_{\mathrm{Pet}, r}\right), \Delta_{\mathrm{SG}}\left(\Theta_{\mathrm{e}}, m_{\mathrm{Pet}, r}\right)$, and $\Delta_{\mathrm{fib}}\left(\Theta_{\mathrm{e}}, m_{\mathrm{Pet}, r}\right)$ down past the limits of the spectroscopic sample. In this regard, it is significant both that the derived functions are smooth all the way down to the selection limits, and that we do not see obvious cut-offs in the data associated with these limits.

In Figure 2, we show how these selection criteria translate onto the $\left(M_{*}, R_{\mathrm{e}}\right)$ plane for several example redshifts between 0.035 and 0.12 . The thicker, roughly diagonal, long-dashed lines represent the star/galaxy separation criterion; the dotted lines represent the "cross-talk" fiber magnitude selection; the thinner, short-dashed boxes represent the effect of the "saturation" selection against bright, compact objects.

With reference to this figure, let us now consider each of these three selection criteria in turn. First, it turns out that the star/galaxy separation criterion does not play an important role in terms of incompleteness. This is simply due to the fact that the massive galaxies we are interested in are bright enough that they have enough flux in their wings to make them clearly distinguishable from stars, even despite their small intrinsic sizes. In fact, they are so bright that for $z \lesssim 0.05$, they would induce cross-talk in the spectrograph. For $z \lesssim$ 0.035 , many - perhaps even most—of the most massive $\left(M_{*} \gtrsim\right.$ $\left.10^{11.3} M_{\odot}\right)$ red sequence galaxies would not be considered as spectroscopic targets, because of their high surface brightness.

As a specific example, consider a $5 \times 10^{10} M_{\odot}$ galaxy with $R_{\mathrm{e}}=1 \mathrm{kpc}$. Using the empirical relations derived as above, this galaxy would have $\Delta_{\mathrm{SG}}>0.3$ out to $z=0.133$, where it would have an intrinsic size of 0.42 . This actually nearly coincides with the point where the $m_{\mathrm{Pet}, r}<17.88$ selection limit becomes important $(z<0.139)$. This galaxy would have $\Theta_{\text {Pet }}<2^{\prime \prime}$ for $z<0.075$, and $m_{\mathrm{Pet}, r}<15$ for $z<0.037$. Similarly, this galaxy would have $m_{\mathrm{fib}, i}<14.5$ for $z<0.27$. It would thus be excluded as a spectroscopic target for $z \lesssim 0.04$ by the saturation and/or cross-talk selection limits, and for $z \gtrsim 0.12$ by the star/ galaxy separation criterion and/or the magnitude limit.

We stress the fact that incompleteness becomes less of an issue at higher redshifts. For example, any galaxies with $M_{*} \gtrsim 10^{11.3} M_{\odot}$ and $R_{\mathrm{e}} \lesssim 2 \mathrm{kpc}$ would not be selected as spectroscopic targets if placed at $z<0.066$ because of their high surface brightness. This is why we have chosen to focus on the $0.066<z<0.12$ interval; at lower redshifts, this cross-talk selection means that galaxies like those of vD08 and D09 would not be targeted for spectroscopic follow-up. In the $0.066<z<0.12$ regime, it turns out that the most important selection effect is due to the $m_{\mathrm{Pet}, r}<15, \Theta_{\mathrm{Pet}}<2^{\prime \prime}$ saturation criterion.

\subsection{Compact Galaxies at High and Low Redshifts}

In Figure 6, we again show the size-mass relation for our sample of massive, red sequence galaxies at $0.066<z<0.12$, with the exception that we have not plotted those galaxies rejected as described in Section 3.1. Furthermore, in contrast to Figure 2, we have used the selection limits derived in Section 4.1 to estimate the relative completeness of the SDSS spectroscopic sample across the $0.066<z<0.12$ volume; these are shown by the contours. These completeness estimates also include the $m_{\text {Pet }, r}<17.77$ selection limit, which can be seen to affect galaxies with $M_{*} \lesssim 10^{10.6} M_{\odot}$ at the distant end of our redshift window.

For comparison, we have also overplotted the high-redshift samples of vD08 (blue points) and D09 (yellow points). Where we have used size measurements from the $z$ band for the SDSS galaxies, these high-redshift studies use the NICMOS F160W filter, which corresponds to rest frame $r$ at $z=1.6$, moving close to $g$ by $z=2.3$. Locally, the difference between $z$ - and $r$-band measured sizes leads to a slightly different slope to the size-mass relation for red sequence galaxies (a slope of 0.65 , rather than 0.56 ). The $r$ - and $z$-band size-mass relations intersect at around $M_{*} \sim 10^{10} M_{\odot}$; the mean $r$-band size at $M_{*} \sim 10^{11} M_{\odot}$ is $15 \%$ larger than in the $z$ band. That is, by using $z$-band derived effective radii, we are, if anything, 


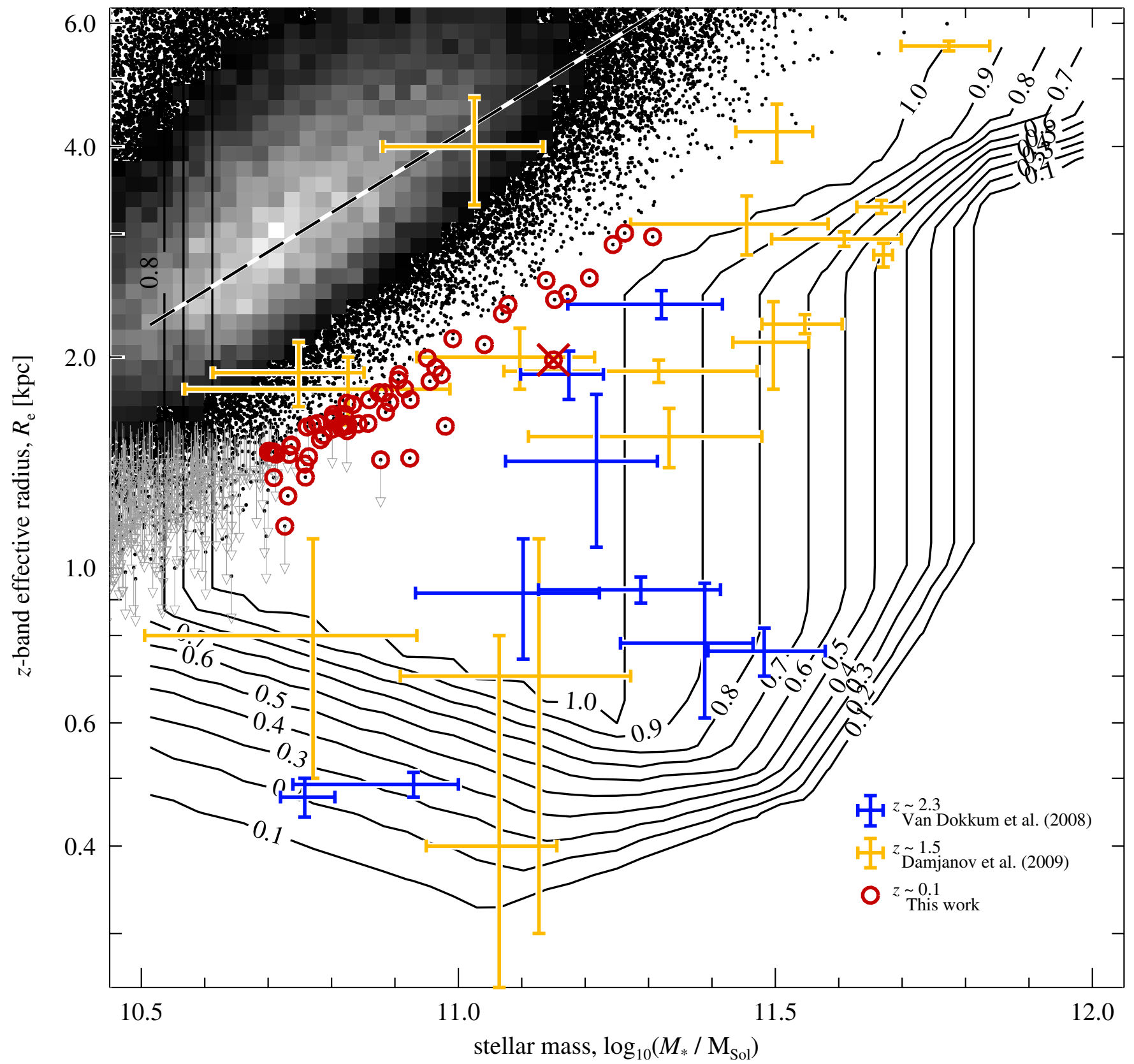

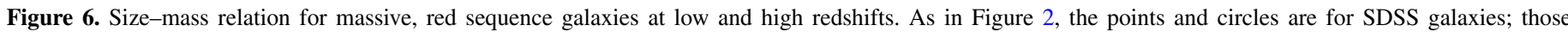

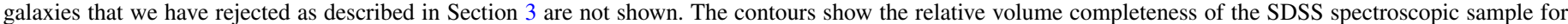

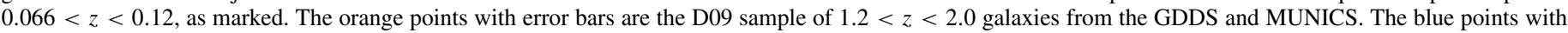

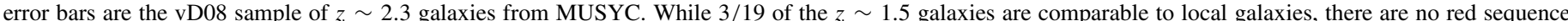

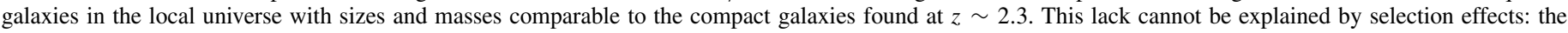

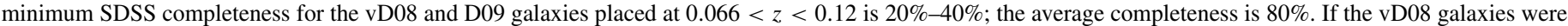
to be passively evolved into the local universe, we would expect to detect on the order of $\sim 6500$ such galaxies.

(A color version of this figure is available in the online journal.)

underestimating the sizes of the local galaxies in comparison to those at high redshift. Similarly, our decision to use the de Vaucouleurs effective radii given in the basic SDSS cata$\log$, rather than the more general Sérsic ones, appears to lead to an underestimate of galaxy sizes. In other words, adopting $r$ - or $g$-band derived sizes, or using Sérsic instead of de Vaucouleurs effective radii, would increase the discrepancy between the high- and low-redshift samples.

One of our candidates (marked with a cross) appears to have properties similar to one of the larger of the vD08 galaxies.
This turns out to be the galaxy whose observed velocity dispersion is inconsistent with its being genuinely compact (Section 3.3). Where we would predict $\Delta \log \sigma=0.24 \mathrm{dex}$, or $\sigma=310 \pm 70 \mathrm{~km} \mathrm{~s}^{-1}$, what we observe is $\Delta \log \sigma=-0.17 \mathrm{dex}$ and $\sigma=129 \pm 14 \mathrm{~km} \mathrm{~s}^{-1}$. This is also the galaxy with the largest difference between the Sérsic and de Vaucouleurs sizes $\left(\log R_{\text {Dev }} / R_{\text {Ser }}=-0.34\right.$; see Section 3.2$)$. Adopting the NYU VAGC Sérsic size measurement is not sufficient to reconcile the observed size and mass with the velocity dispersion: the observed velocity dispersion would still be too 
small by $\sim 0.2$ dex. This galaxy also sits nearly 0.25 dex above the median color-mass-to-light relation shown in Figure 1; using the Bell \& de Jong (2001) prescription for $M_{*} / L$ as a function of $(B-V)$ leads to a stellar mass estimate that is 0.17 dex lower. Adopting both this mass estimate and the NYU VAGC size estimate, we do find consistency between $\Delta \log R_{\mathrm{e}}$ and $\Delta \log \sigma$. In this sense, this galaxy is by far the weakest of our compact galaxy candidates-it seems to have had its size underestimated and/or its mass overestimated.

We also stress that the observed velocity dispersions of the candidates that lie furthest from the main size-mass relation suggest that these galaxies have had their sizes significantly underestimated (see Section 3.3).

It can be seen from Figure 6 that if the vD08 galaxies were placed at $0.066<z<0.12$, the SDSS spectroscopic completeness would typically be $\gtrsim 75 \%$. Note, however, that there are two $R_{\mathrm{e}}<0.5 \mathrm{kpc}$ galaxies from the vD08 sample and one from the D09 sample for which the SDSS completeness is just $20 \%-40 \%$. In Appendix B, we explicitly consider the SDSS spectroscopic completeness for the galaxies in the vD08 sample, if they were to be placed at $z<0.12$. The results presented in this appendix are formally identical to those shown in Figure 6.

If the Kriek et al. (2008b)/vD08 galaxies were not to evolve in either size or number density from $z \sim 2$ to the present day, we would expect there to be $\sim 6500 M_{*}>10^{11} M_{\odot}$ galaxies with $\Delta \log R_{\mathrm{e}}<-0.4 \mathrm{dex}$ at $0.066<z<0.12$, of which $\sim 5250$ should appear in the SDSS spectroscopic sample. Instead, we have only one (very weak) candidate: this galaxy appears to have large errors in its size and mass measurements.

Moreover, we stress that those galaxies which we have identified as "compact" are not qualitatively similar to the compact galaxies found at higher redshifts, which are offset from the local size-mass relation by at least twice as much again as our local compact galaxy candidates.

As an interim conclusion, then, we have shown that there are no galaxies in the local universe (at least as probed by the SDSS spectroscopic sample) that are directly analogous to the compact galaxies found at high redshift. This dearth of compact galaxies cannot be explained by selection effects. In Appendix A, we confirm and extend this conclusion by searching for compact galaxy candidates from within the SDSS photometric sample, on the basis of photometric redshifts.

\subsection{The Number Density of Massive, Compact Galaxies}

In Figure 7, we provide a more quantitative statement of our conclusion with respect to the size evolution of massive galaxies from $z \sim 2$ to $z \sim 0.1$ by plotting the size distribution for massive, red galaxies in different mass bins. In this figure, the filled histograms represent the main SDSS spectroscopic sample described above. For comparison, the horizontal-hatched histograms show the situation at $z \sim 1.6$, based on the ten D09 galaxies drawn from the GDDS; similarly, the diagonal-hatched histograms show the nine $z \sim 2.3$ Kriek et al. (2008b) galaxies with sizes from vD08.

The Kriek et al. (2008b)/vD08 sample is representative, but not complete. In order to derive the densities shown in Figure 7, we have scaled each of the vD08 galaxies as follows. First, we have normalized the distribution to have a density of $1.5 \times$ $10^{-4} \mathrm{Mpc}^{-3}$; this corresponds to the total number density of all $2<z<3$ galaxies to the mass limit of Kriek et al. (2008b), derived from the mass function fit given by Marchesini et al. (2008). We have then scaled this distribution by a factor of $16 / 36$ to count only those galaxies with little or no ongoing star

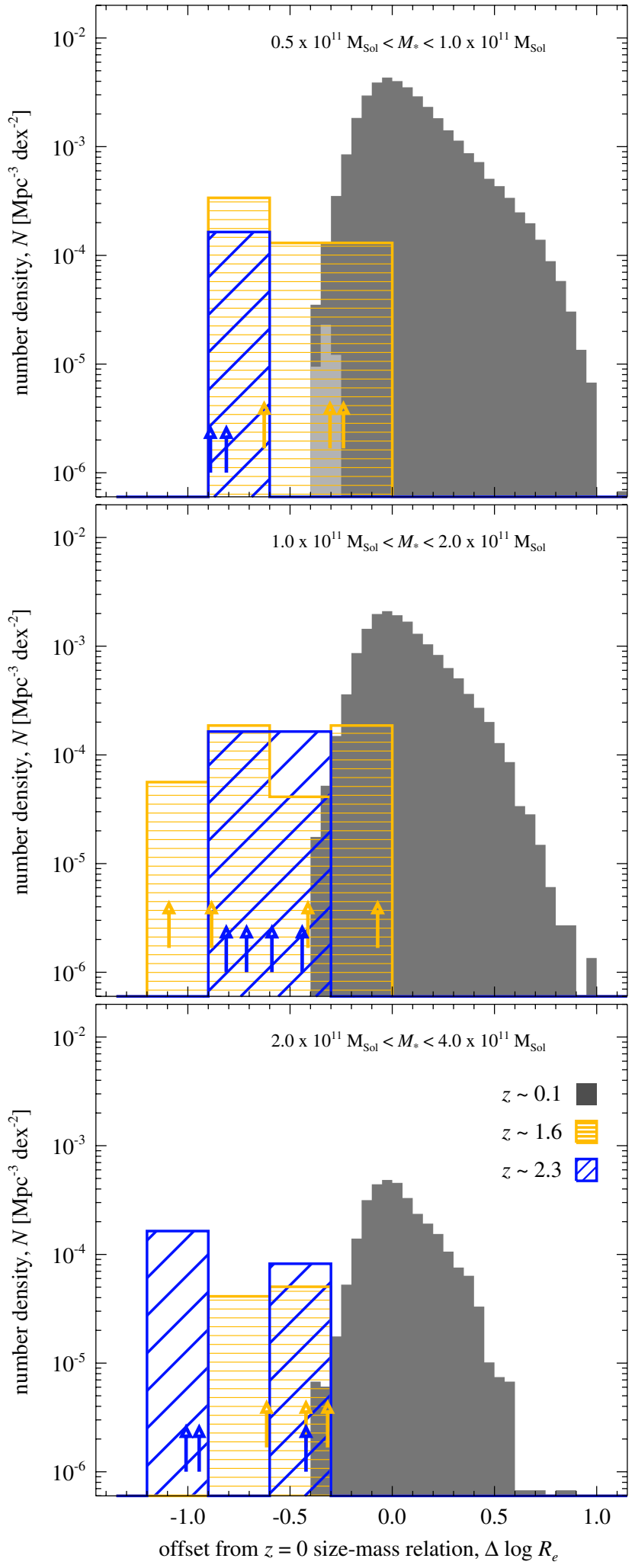

Figure 7. Observed size distribution of massive, red galaxies at $z \sim 0.1, z \sim 1.6$, and $z \sim 2.3$. Each panel of this figure is for a different mass range, as marked. Within each panel, the solid histogram represents the SDSS spectroscopic sample. The blue, diagonally hatched histogram represents the vD08 sample of nine massive, passive galaxies at $z \sim 2.3$; the yellow, horizontally hatched histogram represents the $10 z \sim 1.6$ GDDS galaxies from D09. The arrows at the bottom of each panel indicate the positions of the individual high-redshift galaxies. The $z \sim 2.3$ galaxies have to undergo significant structural evolution over $z \lesssim 2.3$ to match the properties of local universe galaxies; at least part of this evolution has already occurred by $z \sim 1.6$.

(A color version of this figure is available in the online journal.) 


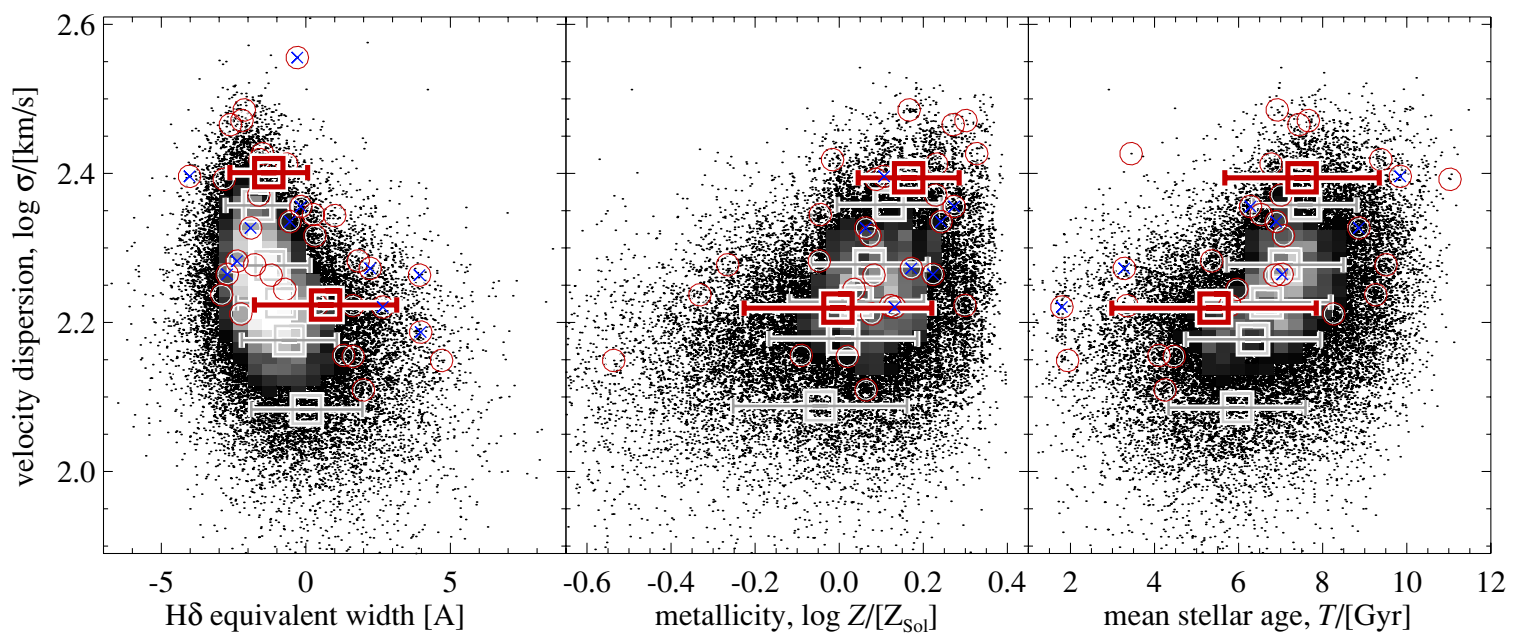

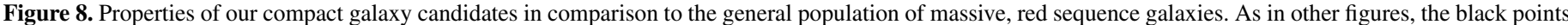

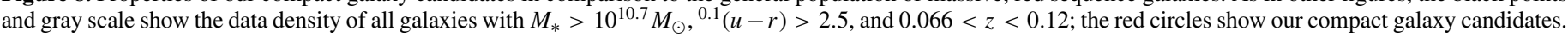

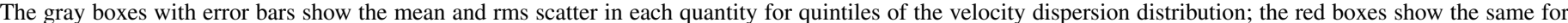

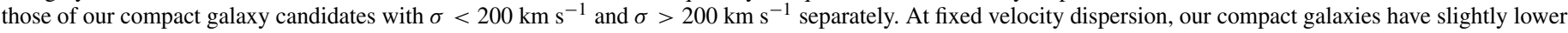
than average mean ages and slightly higher metallicities; however, this result is only significant at the $2 \sigma$ level.

(A color version of this figure is available in the online journal.)

formation from Kriek et al. (2008b) that seem to form a red sequence (Kriek et al. 2008a). For the D09 sample, we are able to use $1 / V_{\max }$ scalings from Glazebrook et al. (2004).

The location of each individual high-redshift galaxy is marked in Figure 7 with an arrow: the slightly lower blue arrows show the vD08 galaxies; the slightly higher yellow arrows are for the D09 galaxies. Clearly, given the small numbers, the uncertainties on these high-redshift values are quite large; even so, they do provide a useful order of magnitude estimate for comparison to the local values.

The clear implication from the comparison between the $z \sim 0.1$ and $z \sim 2.3$ data in Figure 7 is that - in line with the conclusions of vD08 - not one of the vD08 galaxies is consistent with the properties of the $z \sim 0$ galaxy population. With the results we have now presented, we can extend this conclusion by confirming that this discrepancy cannot be explained by selection effects in the low-redshift sample. There are local analogs for less than half of the $z \sim 1.6$ galaxies, albeit with considerably higher number densities. This would imply that at least some $(\lesssim 50 \%)$ of the $z \lesssim 2.3$ evolution has already occurred by $z \sim 1.6$.

\section{DISCUSSION}

\subsection{Compact Galaxy Properties}

In Figure 8, we plot the properties of our compact galaxies in comparison to the general massive, red galaxy population. In each panel, the circles highlight our compact galaxies, while the points and gray scale show all galaxies with $M_{*}>10^{10.7} M_{\odot}$, $0.1(u-r)>2.5$, and $0.066<z<0.12$. The large gray boxes with error bars show the mean and standard deviation of each plotted property in quintiles of the velocity dispersion distribution. Similarly, the red boxes with error bars show the mean and standard deviations for our compact galaxy candidates in two bins, separated at $\sigma=200 \mathrm{~km} \mathrm{~s}^{-1}$, the median for this sample.

In each of the panels of Figure 8 (from left to right), we show the equivalent width of the $\mathrm{H} \delta$ line (where negative values imply emission), the luminosity-weighted mean stellar metallicity, and the luminosity-weighted mean stellar age, as derived by Gallazzi et al. (2005). Because these estimates are available only for DR4, only around half of our compact candidates, and only $3 / 10$ of those with $M_{*}>10^{11} M_{\odot}$, can be plotted in this figure.

We have also matched our compact galaxy sample to the AGN sample described by Kauffmann et al. (2003b), for SDSS DR4. These AGN hosts have been selected by their $[\mathrm{O}$ III $] / \mathrm{H} \beta$ and $[\mathrm{N}$ II] $/ \mathrm{H} \alpha$ emission line ratios, i.e., the Baldwin et al. (1981; BPT) diagram. Thirty-four of our sixty-three galaxies appear in the DR4 catalog; of these, eleven are classified as AGN on the basis of their emission line ratios. This is slightly higher than the AGN fraction of the parent sample, which is in the range $20 \%-26 \%$ for the mass range we are considering. Of the eleven galaxies identified as AGN hosts, five sit on or slightly above the main $M_{*}-L_{[\mathrm{O} \text { III] }}$ relation, with $L_{[\mathrm{O} \text { III] }} \approx 10^{6} L_{\odot}$, four have $L_{[\mathrm{O} \text { III] }} \sim 10^{7-8} L_{\odot}$, and one is quite high luminosity, with $L_{\text {[O } \mathrm{III}]}=10^{8.7} L_{\odot}$. These 11 galaxies are marked in each panel of Figure 8 with a small blue cross.

Kauffmann et al. (2003b) also provide revised stellar mass and velocity dispersion measurements for these AGN-host galaxies. Accounting for the presence of an AGN does not have a major impact on these measurements: the masses and velocity dispersions change at the level of $0.05 \mathrm{dex}$ and $16 \mathrm{~km} \mathrm{~s}^{-1}$, respectively. That is, while it is possible that an optically bright point source may bias the measured sizes of these galaxies downward, within the stated errors, the AGN does not significantly affect the derived values of $M_{*}$ or $\sigma$. (It is relevant here that only one of our compact galaxy candidates shows a significant residual point source after subtracting off the best-fit Sérsic profile, as produced by GALFIT; see Section 3.2.)

Looking now at Figure 8, it is clear that the majority of our compact galaxy candidates have quite old stellar populations. For the $\sigma>200 \mathrm{~km} \mathrm{~s}^{-1}$ bin, all but one of our candidates have $T>6$ Gyr. For the $\sigma<200 \mathrm{~km} \mathrm{~s}^{-1}$ galaxies, the median age is $6 \mathrm{Gyr}$ (although the ages do range from 2 to $10 \mathrm{Gyr}$ ). Among the lower velocity dispersion candidates, there is a clear tendency toward relatively high equivalent widths for $\mathrm{H} \delta$ absorption, suggestive of a relatively recent $(\lesssim 2$ Gyr $)$ star formation event. That is, at fixed velocity 
dispersion, our compact galaxy candidates appear to have slightly higher metallicities, and be slightly younger than average.

That said, using bootstrap resampling on similar sized samples drawn randomly from the mass-limited sample, and controlling for velocity dispersion, these results are weakly significant at best: $1.9 \sigma$ for the age, and $<1 \sigma$ for metallicity. Considering only the higher velocity dispersion candidates $\left(\sigma>200 \mathrm{~km} \mathrm{~s}^{-1}\right)$, the significance of these differences becomes $1.9 \sigma$ and $2.2 \sigma$ for the age and metallicity offsets, respectively. This weakly significant result should be contrasted with the results of Shankar \& Bernardi (2009) and van der Wel et al. (2009), who find that, on average and at fixed dynamical mass, earlytype galaxies with higher velocity dispersions (or, equivalently, smaller sizes) have older mean stellar ages.

While the younger mean stellar ages and lower metallicities of our compact galaxy sample are only weakly significant, both would imply a relatively late start to star formation for these galaxies and/or their progenitors. But if these galaxies grow in size through mergers (for example) then it is possible that these galaxies are small not because their formation is delayed relative to other galaxies of the same mass or velocity dispersion, but rather because they have had fewer mergers overall, or perhaps just fewer recent mergers. That is, it may be that, at fixed mass, these compact galaxies are in fact older, in the sense that they have been assembled earlier, and existed in (more or less) their present form for longer than other galaxies of the same mass or velocity dispersion.

\subsection{Comparison to Other Recent Works}

In a similar study to this, using sizes and photometry from the NYU VAGC for SDSS DR6, Trujillo et al. (2009, hereafter T09) have recently reported the detection of 28 compact galaxies at $z<0.2$. These galaxies were selected to have $M_{*}>8 \times 10^{10} M_{\odot}$ and $R_{\mathrm{e}}<1.5 \mathrm{kpc}$. To the same size and mass limits, we find just one galaxy in our $0.066<z<0.12$ red sequence sample. This implies a difference in volume density of a factor of 5.5. Whereas we are primarily interested in passive (red sequence) galaxies, T09 did not preselect their compact galaxy candidates by color. Applying our ${ }^{0.1}(u-r)>2.5$ criterion to the T09 sample, only $30 \%(9 / 28)$ of these galaxies are red. In other words, to the same limits, our results are in good agreement with those of T09 for red galaxies: based on the single galaxy in our sample that satisfies the T09 size-mass selection, our inferred number densities agree to within $65 \%$.

At the same time, however, T09 find a number of blue compact galaxies while we do not. If we expand our sample to include blue galaxies, after visual inspection of an additional 34 objects, we are left with only 7 plausible blue compact galaxy candidates. (Note that none of these galaxies have masses greater than $8 \times 10^{10} M_{\odot}$.) In an attempt to better understand the cause of this discrepancy, we have compared the $r$-band model effective radii from the basic SDSS catalog to the $r$-band Sérsic-fit sizes from the NYU VAGC that are used by T09. For all four of the galaxies in the T09 sample that lie at $z<0.14$, the agreement is better than $25 \%$. For the galaxies at $z>0.14$, however, the agreement becomes progressively worse; the median NYU VAGC size is approximately $40 \%$ larger than that given in the basic SDSS catalog. The random scatter between the two size measurements does not obviously depend on the observed sizes, but does appear to be significantly higher for $m_{r} \gtrsim 17$; all of the $z<0.14$ T09 galaxies have $m_{r}<17$. This suggests that the T09 size measurements may be affected by larger random or systematic size measurement errors at higher redshifts, due to lower signal to noise. We also note that each and every one of the T09 galaxies has an apparent size smaller than our conservative upper limit of 0.75 . Follow-up observations are required to robustly determine the sizes of these galaxies.

We have also compared our mass estimates for the T09 sample to those given in the NYU VAGC and used by T09. We find systematically lower masses for the galaxies in the T09 galaxies, at the level of $0.05-0.10$ dex. Interestingly, all but one of the T09 galaxies lie below the median color- $(M / L)$ relation shown in Figure 1. (The exception lies 0.16 dex above it.)

There are thus significant systematic differences between both the size and mass estimates used here, and those used by T09. Without follow-up observations, however, it is not possible to make any further conclusions-we are, after all, analyzing the same data. That said, we stress the fact that the observed velocity dispersions for our candidates with $\Delta \log R_{\mathrm{e}}<-0.4$ dex imply that their very small inferred sizes are produced by large errors in the measured sizes (see Section 3.3). In contrast to this, T09 find a median velocity dispersion than is only 0.04 dex higher than their control sample, even though the mean size and mass are offset from the Shen et al. (2003) relation by -0.5 dex. This discrepancy can only be explained by either very large structural differences, or if the T09 sample is disproportionally affected by large measurement errors in size and/or mass. Spatially resolved dynamical studies of the two samples may offer a practical means of resolving this issue.

While this discrepancy in the numbers of blue compact galaxies remains a concern, it has no impact on our conclusions. As well as being blue, the T09 galaxies have mean stellar ages of $\lesssim 2 \mathrm{Gyr}$; that is, their current ages are comparable to the ages of the vD08 galaxies at $z \gtrsim 2$. In this sense, the T09 sample may represent close analogs to the vD08 and D09 galaxies, but they cannot be the passively evolved counterparts to the galaxies seen at $z \gtrsim 2$ that we are interested in here.

Even more recently, Valentinuzzi et al. (2010, hereafter V10) have described a sample of 122 compact galaxies selected from the WIde-field Nearby Galaxy-cluster Survey (WINGS) of $\mathrm{X}$-ray selected clusters at $0.04<z<0.07$. V10 compared their mass estimates to the ones that we use for this paper, and found an offset of 0.09 dex. After scaling our masses up to account for this offset, however, the median size-mass relation for our sample is significantly offset from the one given by V10; the median offset is 0.14 dex, with the V10 values being smaller than ours. This is despite the fact that V10 find no systematic difference between their sizes and the sizes that we use here. The cause of this offset thus remains unclear, but we consider it unlikely to be due to environmental effects - a number of studies (e.g., Kauffmann et al. 2004; Park et al. 2007; Weinmann et al. 2009) have found little or no evidence for a strong environmental dependence of the size-mass relation among SDSS galaxies.

V10 find that $22 \%$ of their sample of cluster galaxies with $10.5<\log \left(M_{*} / M_{\odot}\right)<11.6$ satisfy their "superdense" criterion of $\Sigma_{\mathrm{e}}=M_{*} / 2 \pi R_{\mathrm{e}}^{2}>10^{9.5} M_{\odot} \mathrm{kpc}^{-2}$. If we apply the V10 criterion to our red sequence galaxy sample (scaling our masses by 0.09 dex), we find that only $1.1 \%$ of the $10.5<$ $\log \left(M_{*} / M_{\odot}\right)<11.6$ galaxies satisfy the V10 "superdense" criterion; in the same mass range, the fraction for red sequence galaxies is $1.3 \%$. This is substantially different from their value of $22 \%$. If we then scale our sizes down by 0.14 dex so that our size-mass relation matches the V10 one, our fractions increase to $11 \%$ and $16 \%$. Hence, the results are compatible, but only 
after they have been scaled to the same mass-size relation. In effect, this implies that observing the fraction of outliers defined as outliers with regards to the mass size relation, as we have done in this paper, is stable. We have not been able to identify the cause of the discrepancies in the mass-size relation between the two samples. More direct comparisons of galaxies measured by SDSS and V10, as well as parallel testing of the different measurements algorithms, would help to resolve these issues.

Finally, we stress that according to both our results and those of V10, the vD08 galaxies must undergo significant size evolution. For the vD08 galaxies, the median size offset from the V10 size-mass relation is $-0.68 \mathrm{dex}$, or a factor of 5. The median offset from our own size-mass relation is -0.90 dex. In either case, this offset is larger than even the most extreme outliers at $z \approx 0$. Accepting the vD08 results, the high-redshift galaxies, as a population, simply cannot evolve passively without structural evolution into the red sequence and/ or early-type galaxies found in the local universe.

\section{SUMMARY AND CONCLUSIONS}

The central question of this work has been the existence (or non-existence) of massive, compact, quiescent, and/or early-type galaxies in the local universe, and particularly the importance of selection effects in the SDSS spectroscopic sample for such galaxies. We have shown that, especially for lower redshifts $(z \lesssim 0.05)$, galaxies with the masses and sizes of those found at $z \gtrsim 2$ would not be targeted for spectroscopic follow-up (Figure 2). The main reason for this is not the star/ galaxy separation criterion, but rather the exclusion of bright and compact targets in order to avoid saturation and cross-talk in the spectrograph (see Section 4.1).

Bearing this in mind, we have conducted a search for massive, compact galaxies at $0.066<z<0.12$; we estimate that, in this redshift interval, the average completeness for galaxies like those from vD08 and D09 would be $\gtrsim 20 \%$ in the worst case, and $\sim 80 \%$ on average (Figure 6 ). Starting from a sample of massive $\left(M *>10^{10.7} M_{\odot}\right)$ red sequence $\left({ }^{0.1}(u-r)>2.5\right)$ galaxies, we have selected the 280 galaxies with inferred sizes that are a factor of 2 or more smaller than would be expected from the Shen et al. (2003) $M_{*}-R_{\mathrm{e}}$ relation for early-type galaxies. In order to confirm their photometry and size measurements, we have visually inspected all of these objects. Unsurprisingly, by selecting the most extreme outliers, a large fraction of these objects $(\sim 70 \%)$ appear to be instances where the size and/or stellar mass estimates are unreliable (Section 3.1).

For the 63 galaxies with no obvious reason to suspect their size or stellar mass estimates, there is good agreement between the default SDSS size measurement (based on the twodimensional light distribution, using a sector-fitting algorithm, and assuming a de Vaucouleurs profile), and those given in the NYU VAGC (based on the azimuthally average growth curve, assuming a more general Sérsic profile). However, particularly for galaxies with high $n$, the de Vaucouleurs size measurement is systematically smaller than the Sérsic one, at the level of $\lesssim 25 \%$ (Section 3.2).

In general, and as expected, our 63 compact galaxy candidates have significantly higher than average velocity dispersions (Figure 5). While it remains possible that the sizes of at least some of our compact galaxy candidates may have had their sizes underestimated by $\sim 30 \%$, in general, the relatively high observed velocity dispersions support the notion that they are indeed unusually compact given their stellar masses (Section 3.3).
Among our compact galaxy candidates, there are no galaxies with sizes comparable to those found at $z \sim 2.3$ by vD08; we find analogs for $\lesssim 50 \%$ of the D09 galaxies at $z \sim 1.6$ (Figures 6 and 7). This lack cannot be explained by selection effects: if the vD08 galaxies were passively evolved into the local universe, we would expect to detect on the order of $\gtrsim 5000$ galaxies in this region of the size-mass diagram.

To test this conclusion, in Appendix A, we have also used photometric redshifts to construct the size-mass diagram for the full photometric sample, and compared this to the size-mass diagram for the spectroscopic subsample, also analyzed using photometric redshifts. In this way, we can also make an estimate for the number of massive, compact galaxies that are missing from the spectroscopic sample. While it is conceivable that SDSS is missing (at most) a handful of massive, compact galaxies, there are again no signs of galaxies comparable to those of vD08 or D09.

It is not impossible that some systematic errors in the estimation of $M_{*} / L s$ for the high-redshift galaxies (e.g., an evolving stellar IMF) mean that their stellar masses are vastly overestimated; however, it would require an overestimate of $\gtrsim 0.7$ dex to reconcile the vD08 galaxies with the sizes of the smallest galaxies we have identified in the SDSS catalog. Further, we stress that we have verified that the masses of the $z \sim 0$ and $z \sim 2.3$ galaxies have been derived consistently. By the same token, there may be systematic errors in the measured sizes of the high- and low- $z$ galaxies. vD08 point out that complex morphologies, and in particular the possibility of low surface brightness wings, could mean that the sizes of the $z \sim 2.3$ galaxies are underestimated. We point out, however, that all indications are that the sizes that we have used here are underestimates; using different size measures for the low- $z$ galaxies would only increase the discrepancy between the highand low-redshift samples, so strengthening our conclusions.

Accepting the high-redshift observations at face value, then, our results confirm that massive galaxies, both individually and as a population, must undergo considerable structural evolution over the interval $z \lesssim 2.3$ in order to develop into the kinds of galaxies seen locally — even after star formation in these galaxies has effectively ended. We see some hints that a significant amount of this evolution $(\lesssim 50 \%)$ may have already occurred by $z \sim 1.6$.

The fact that each and every one of the vD08 galaxies must undergo significant structural evolution to match the properties of present-day galaxies implies that the mechanism that drives this growth must apply more or less evenly to all galaxies. To see this, let us assume that some external process drives the size evolution of these galaxies, and that even a single event is sufficient to move an individual galaxy onto the main size-mass relation. Then, we can assume some simple probability distribution for the number of events, $N$, among individual galaxies. (For example, we could assume that events occur randomly across the time interval $z<2.3$, or that each galaxy experiences $N \pm \sqrt{N}$ events.) Now, our results suggest that the number density of vD08-like galaxies drops by at least a factor of 5000 since $z \sim 2.3$. In order to ensure that at most $1 / 5000$ galaxies have $N=0$ after $z \sim 2.3$, simple probabilistic arguments imply that the average galaxy must undergo $\gtrsim 20$ events. This would imply that a strongly stochastic process like major mergers cannot be the primary mechanism for the strong size evolution of massive galaxies.

Apart from their small sizes and high velocity dispersions, our compact galaxy candidates are not obviously distinct from 

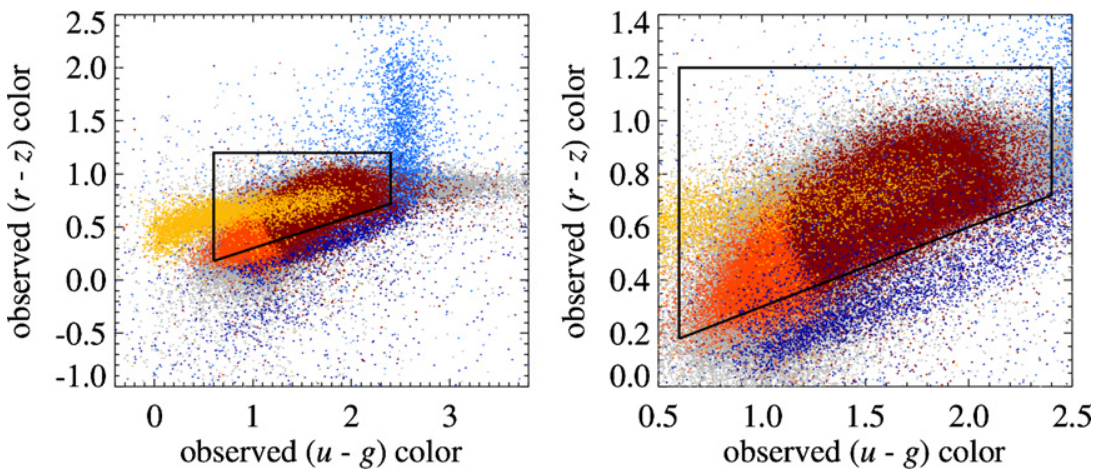

Figure 9. Selecting $z \lesssim 0.1$ galaxies based on color alone. Each panel shows the observed $(u-g)-(r-z)$ color-color plot for objects in the SDSS spectroscopic sample; the right panel simply shows the central region in greater detail. Points are color coded according to their spectral classification, viz.: galaxies (gray), galaxies with $0.066<z<0.10$ (light red), galaxies with $0.066<z<0.10$ and ${ }^{0.1}(u-r)>2.5$ (dark red), quasars (yellow), late-type stars (light blue), and ordinary stars (dark blue). The box shows the color selection that we use to select $z \lesssim 0.1$ galaxies. This selection should produce a reasonably complete sample of $z \lesssim 0.1$ galaxies, with some contamination from both stars and quasars. In particular, later-type stars and some quasars have observed SEDs that are very similar to red sequence galaxies at $z \sim 0.1$.

(A color version of this figure is available in the online journal.)

the general population (Figure 8). If anything, at fixed velocity dispersion, our compact galaxies have stellar populations that are slightly younger than average (at $\sim 1.9 \sigma$ significance). Even so, the majority of these galaxies' stellar populations are definitely "old," with luminosity-weighted mean stellar ages typically in the range 6-10 Gyr. But if some external mechanism drives the size evolution of these galaxies, we speculate that their small sizes may indicate that they have assumed their present form comparatively early, and in this sense they may actually be relatively old (see also, e.g., van der Wel et al. 2009). If so, with better understanding of the processes that determine the sizes of early-type galaxies, and in particular the role of merging, the properties of these galaxies could provide a means of constraining the evolution of massive galaxies after they have completed their star formation, including their late-time merger histories.

We thank both Tiziano Valentinuzzi and Ignacio Trujillo for helpful discussions in trying to reconcile our results. This work was supported through grants by the Nederlandse Organisatie voor Wetenschappelijk Onderzoek (NWO) and the Leids Kerkhoven-Bosscha Fonds (LKBF).

\section{APPENDIX A}

\section{LOOKING FOR MASSIVE COMPACT GALAXIES IN THE SDSS PHOTOMETRIC SAMPLE}

In this appendix, we present a complementary analysis in which we directly compare the spectroscopic and photometric SDSS samples, in order to test our conclusion that the apparent lack of massive, compact galaxies in the local universe cannot be explained by incompleteness in the spectroscopic sample.

\section{A.1. Selecting Galaxies by Color Alone}

Before we can address the question of massive compact galaxies in the SDSS photometric sample, we must first devise a means of separating stars and galaxies without selecting on the basis of observed size or light profile. Our method for doing so is shown in Figure 9, which plots the observed (extinction-corrected) ugrz colors of different classes of objects from the spectroscopic sample; we show all galaxies (gray), $0.066<z<0.12$ galaxies (bright red), and those with $0.1(u-r)>2.5$ (dark red), O-K stars (dark blue), M-type or later stars (light blue), and quasars (yellow).

The black box shown in Figure 9 shows our criteria for selecting $0.066<z<0.12$ galaxies based on their ugrz colors:

$$
0.6<(u-g)<2.4 \text { and } 0.3 \times(u-g)<(r-z)<1.2 \text {. }
$$

Again, we apply this selection in terms of model colors. Note how, whereas the stellar sequence is reasonably well separated from the region of color space occupied by galaxies for $(u-g) \lesssim 2.5$, beyond this point, the late-type stellar sequence turns up, such that late-type stars and galaxies are blended. In the most general terms possible, the mean galaxy redshift increases toward redder $(u-g)$ colors. This means that our ability to distinguish red galaxies from late-type stars on the basis of their optical SEDs is limited to $z \lesssim 0.12$.

In the right-hand panel of Figure 9, we zoom in on this selection region. From this panel, it is clear that a large proportion of quasars will also be included in our color-selected "galaxy" sample. Similarly, it is clear that this color selection is not $100 \%$ efficient in excluding stars from our sample: more quantitatively, with this selection we are able to exclude more than $80 \%$ of the spectroscopically identified stars that are given $0.066<z_{\text {phot }}<0.12$, while retaining more than $97 \%$ of all spectroscopically targeted and confirmed galaxies that are given $0.066<z_{\text {phot }}<0.12$. Furthermore, it should be remembered that stars are already heavily selected against for the spectroscopic sample plotted in Figure 9; the relative number of stellar "contaminants" may well be considerably higher for the photometric sample.

\section{A.2. Photometric Redshifts and Stellar Mass Estimates}

A major improvement in DR7 is a complete revision in how the basic (photoz) photometric redshifts are derived (Abazajian et al. 2009). Rather than using some combination of synthetic template spectra to fit galaxies' SEDs, the new photoz algorithm directly compares the observed colors of individual galaxies to those of a reference sample of galaxies with spectroscopic redshifts. Specifically, for each individual object, the algorithm finds the 100 closest neighbors in ugriz color space, and fits a hyper-plane to these points, rejecting outliers; the photoz is then determined by interpolating along 
this 4D surface. In comparison to the DR6 algorithm, this reduces the RMS redshift error by more than $75 \%(\langle\Delta z\rangle=$ 0.025 ), and significantly reduces systematic errors (Abazajian et al. 2009).

For this exercise, rather than derive SED-fit stellar mass estimates assuming the photometric redshifts, we will simply use the empirical relation between ${ }^{0.1}(g-i)$ color and $M_{*} / L$ (Equation (1)). In this way, we are able to recover the $z_{\text {spec }}{ }^{-}$ derived, SED-fit $M_{*} / L \mathrm{~s}$ of the sample of galaxies shown in Figure 1 to $0.045 \operatorname{dex}(1 \sigma)$; including the effects of photometric redshift errors, $k$-corrections, and $M_{*} / L$ errors, the total $(1 \sigma)$ error in $M_{*}$ is 0.13 dex. This should be compared to the median formal error on the original SED-fit stellar mass estimates $(0.10 \mathrm{dex})$. That is, the errors on $M_{*}$ based on photometric masses (adding these two errors in quadrature) are only about $60 \%$ greater than those based on spectroscopic redshifts.

\section{A.3. The Size Distribution of Massive, Red Sequence Galaxies}

In Figure 10, we show three size-mass diagrams corresponding to, from top to bottom: (a) the spectroscopic sample, analyzed using spectroscopic redshifts; (b) the spectroscopic sample, analyzed using photometric redshifts; and (c) the photometric sample, analyzed using photometric redshifts. In all three cases, the only selections applied to each sample are on photometric type (to exclude optical artifacts, etc., we require either a star or galaxy type classification) and $u g r z$ color (to exclude stars); then, as in Figures 2 and 6, we are only showing those galaxies inferred to have $0.066<z<0.12$ and $0.1(u-r)>2.5$. Again, objects with measured sizes smaller than $0^{\prime} .75$ are shown as upper limits, assuming a size of 0.75 . When comparing these three different analyses, the difference between (a) and (b) shows the effect of using spectroscopic versus photometric redshifts, and the difference between (b) and (c) shows the difference between the SDSS spectroscopic and photometric selection. That is, the comparison between (b) and (c) gives a direct indication of the level of incompleteness in the spectroscopic sample.

Looking at panels (a) and (b), it is clear that the use of photometric redshifts produces a considerably greater scatter in the size-mass diagram, including a rather large number of galaxies with inferred stellar masses of $10^{12} M_{\odot}$ or greater. There is a clear excess of unresolved objects with inferred stellar masses greater than $\sim 10^{11} M_{\odot}$ in panel (b) in comparison to panel (a) However, we already know from Section 3 that there are no objects in the spectroscopic sample with these sizes and masses-the unresolved objects in panel (b) cannot be genuine compact galaxies. Of the 34 with inferred $M_{*}>10^{11} M_{\odot}, 16$ of these objects are spectrally identified as being stars, and 1 as a quasar at $z=0.102$. Of the 17 spectrally confirmed galaxies, all have $\left|z_{\text {phot }}-z_{\text {spec }}\right| \gtrsim 0.02$. Of these, 15 have had their redshifts, and thus stellar masses, seriously overestimated; the other two are at $z>0.12$, and so have had their intrinsic sizes underestimated.

Turning now to the comparison between panels (b) and (c), the first point to make is that the excess of unresolved sources is even more pronounced. We have matched all of these objects to the Two Micron All Sky Survey (2MASS; see Cutri et al. 2003 and Skrutskie et al. 2006) point source catalog in order to investigate their NIR colors. $90 \%$ of these objects fall in the stellar region of the $(J-K)-K$ color-magnitude plot; similarly, $80 \%$ fall in the stellar region of a $(g-z)-(J-K)$ color-color plot.
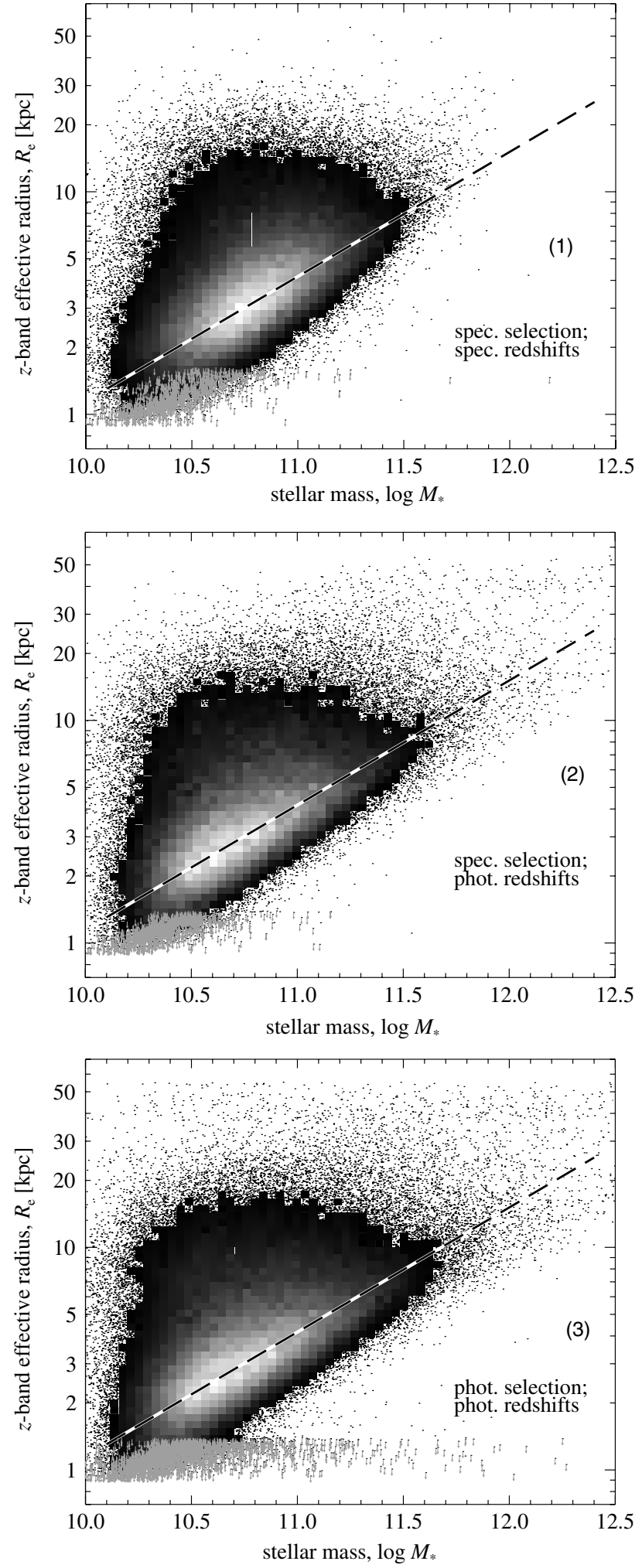

Figure 10. Size-mass plot for massive, red sequence galaxies at $0.066<z<$ 0.12 , based on the spectroscopic and the full photometric SDSS catalogs. Each panel shows the sizes and masses of galaxies based on, from top to bottom, the spectroscopic sample using spectroscopic redshifts, the spectroscopic sample using photometric redshifts, and the photometric sample using photometric redshifts; in each case, only those objects inferred to have ${ }^{0.1}(u-r)>2.5$ and $0.066<z<0.10$ are shown. In panel 3, many more objects with inferred sizes $\lesssim 0.3 \mathrm{kpc}$ can be seen; these are largely stars misclassified (in terms of their photometric redshifts) as galaxies. For $M_{*} \lesssim 10^{10.8} M_{\odot}$ and $R_{\mathrm{e}} \lesssim 10^{-0.2} \mathrm{kpc}$, comparison between panels 2 and 3 suggest that there may be a few additional galaxies in the photometric sample that do not appear in the spectroscopic sample. 
Further, we have visually inspected the 434 objects with inferred $M_{*}>10^{11} M_{\odot}$ and with sizes smaller than the main $M_{*}-R_{\mathrm{e}}$ relation by 0.4 dex or more. Roughly $70 \%$ of these objects are obviously stars: 133 come from crowded Galactic fields covered as part of SEGUE; 126 are double stars; 49 have clear diffraction spikes and/or are clearly saturated. Another 12 objects have been cross-matched with the USNO-B star catalog (within $1^{\prime \prime}$ ), and have measured proper motions of $1^{\prime \prime}-4^{\prime \prime} \mathrm{yr}^{-1}$. Nineteen objects are the central point sources of very large spiral galaxies; most of these are also found in the ROSAT and/ or FIRST catalogs. We also note that there are 17 very small disk or irregular galaxies with red point sources at or very near their centers. Most of these also have proper motion measurements from the USNO-B catalog, and several are spectrally identified as late-type stars; it seems plausible that these galaxies simply have foreground stars coincidentally superposed very near their centers.

In short, of the 434 objects from the full photometric sample that, on the basis of photometric redshifts, are inferred to have $M_{*}>10^{11} M_{\odot}$ and $\Delta R_{\mathrm{e}}<-0.4 \mathrm{dex}$, not one remains as a viable compact galaxy candidate.

\section{A.4. Estimating the Importance of Spectroscopic Selection Effects}

The conclusion from both the analyses that we have now presented is that there are no galaxies in the local universe with sizes and masses comparable to the compact galaxies found at higher redshifts. In Figure 11, we provide a more quantitative statement of this conclusion, by plotting the size distribution for massive, red galaxies in different mass bins.

In this figure, the filled histograms represent the main SDSS spectroscopic sample, analyzed using spectroscopic redshifts, as in Section 3. The heavy black and red histograms represent the spectroscopic and photometric samples, respectively, analyzed using photometric redshifts, as in Section A.3. In all cases, objects excluded on the basis of visual inspection are not plotted; this accounts for the sharp cutoffs at $\Delta \log R_{\mathrm{e}}=-0.3$ and at $\Delta \log R_{\mathrm{e}}=-0.4$ for the filled and open histograms, respectively. Immediately above these cutoffs, where we have done no visual inspection but where there is likely to still be significant contamination, these distributions should be regarded as upper limits on the true distribution. In the upper panel, we plot those objects with observed sizes smaller than 0.75 separately as the light gray filled histogram, and the thin black and red histograms.

As in Section A.3, the difference between the filled and solid black histogram, both of which are derived from the spectroscopic sample, shows the increased scatter due to the use of photometric redshifts. Similarly, the difference between the black and red histograms shows the difference between the spectroscopic and photometric samples, and so allows a quantification of the bias in the spectroscopic sample. By simply tallying the numbers of galaxies with $-0.4<\Delta \log R_{\mathrm{e}}<-0.3$, we find that the "completeness" (the ratio between the number of galaxies in the spectroscopic sample compared to the full photometric sample) is $75 \%, 68 \%, 67 \%$, and $43 \%$ for each of these mass bins, from lowest to highest.

In order to improve on these estimates, we have done the following. Using the approach described above, we have assigned each object a weight according to its $z_{\text {phot }}$-derived mass and size. Then, going back to the spectroscopic sample, we use these to compute the mean weight in cells of $z_{\text {spec }}-$ derived mass and size. The completeness contours we derive in

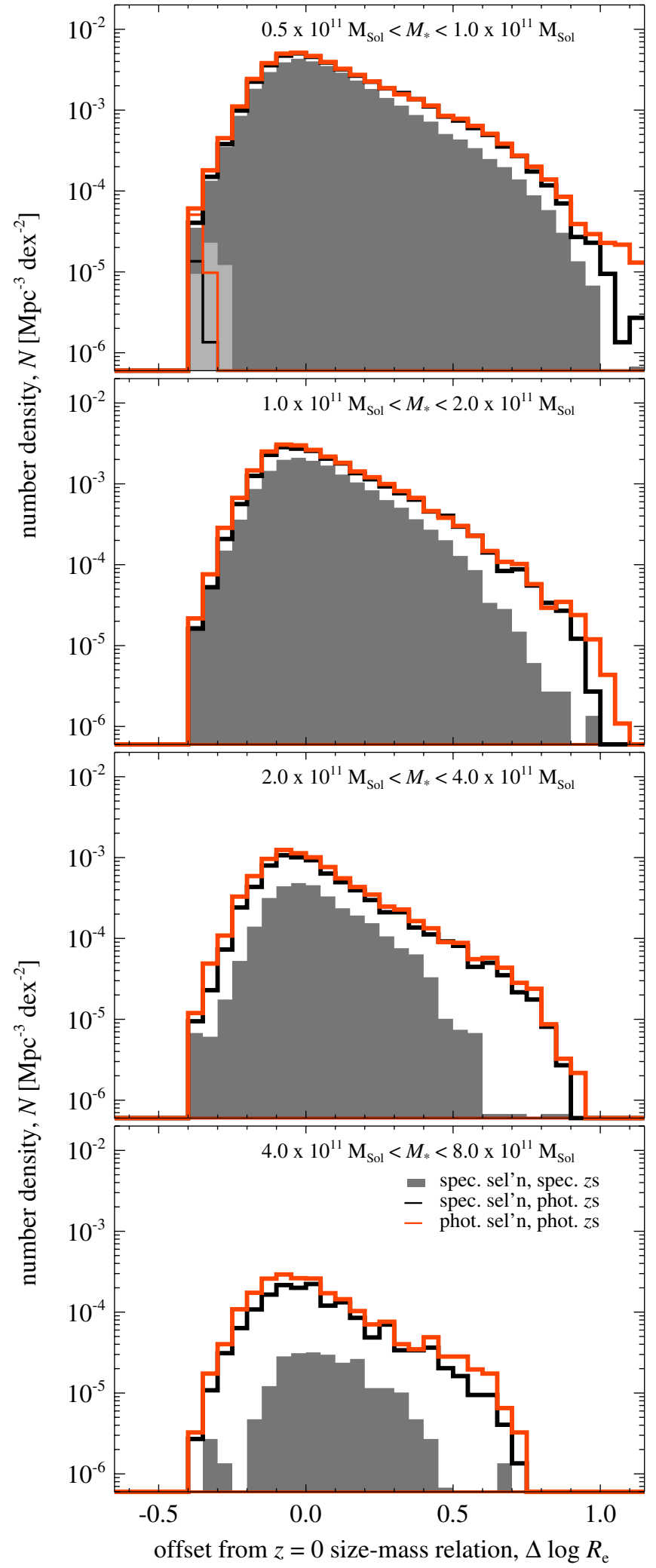

Figure 11. Observed size distribution of massive, red galaxies at $0.066<$ $z<0.12$. Each panel is for a different mass range as marked. In each panel, the solid histogram represents the SDSS spectroscopic sample, analyzed using spectroscopic redshifts. The black and red histograms are the SDSS spectroscopic and photometric samples, respectively, analyzed using photometric redshifts. We have visually inspected all objects with inferred $M_{*}>10^{11} M_{\odot}$ and $\Delta R_{\mathrm{e}}<-0.5 \mathrm{dex}$; not one of these objects is a plausible massive, compact galaxy candidate. The fact that the shape of the red histogram does not differ significantly from that of the black histogram for $\Delta R_{\mathrm{e}}>-0.5$ dex indicates that the spectroscopic sample is not significantly biased against compact galaxies. (A color version of this figure is available in the online journal.) 

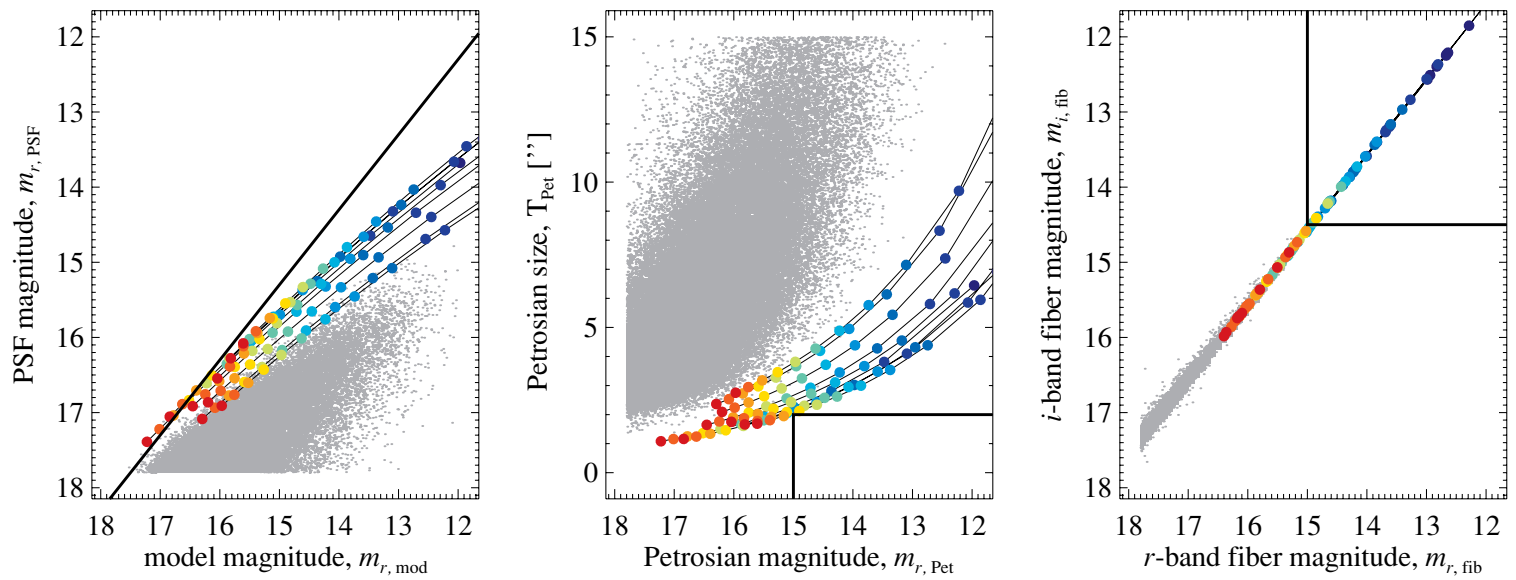

Figure 12. Modeling the selection of the vD08 sample at low redshifts. Each panel shows one of the three important spectroscopic selection criteria described in Section 4.1 (left to right): the star galaxy separation criterion, the saturation criterion, and the fiber cross-talk criterion. The fine gray points show galaxies in our main sample of massive, red sequence galaxies. The tracks show how individual galaxies in the vD08 sample would appear in the SDSS if they were to be placed at low redshift; the large points show the individual galaxies at $z=0.01,0.02, \ldots, 0.12$. In each case, the tracks go from right to left as they go from low- to high redshift. The hatched region shows where galaxies will not be selected as SDSS spectroscopic targets. Incompleteness due to poor star/galaxy separation only becomes a concern for the very smallest and lower mass vD08 galaxies at $z \gtrsim 0.1$. At $z \sim 0.08$, many of the vD08 galaxies would fall very close to the saturation detection limit: this selection is a concern for galaxies slightly smaller and/or brighter than the vD08 galaxies. The most important selection limit is the fiber cross-talk selection: none of the vD08 galaxies would satisfy this selection limit if placed at $z \lesssim 0.04$.

(A color version of this figure is available in the online journal.)

this way are in good qualitative agreement with those shown in Figure 2, although they suggest incompleteness at the $2 \%-5 \%$ level for mean-sized galaxies with $M_{*} \gtrsim 10^{11} M_{\odot}$. Using these values to estimate the number of $M_{*}>10^{11} M_{\odot}$ galaxies with $\Delta R_{\mathrm{e}}<-0.3 \mathrm{dex}$, this suggests that the spectroscopic sample is missing on the order of four such galaxies.

\section{APPENDIX B}

\section{ESTIMATING THE SDSS SPECTROSCOPIC COMPLETENESS FOR GALAXIES IN THE VD08 SAMPLE}

In Section 4.1, we have used our main sample of massive, red sequence galaxies to derive empirical relations between the observed quantities that are important for SDSS spectroscopic target selection like apparent magnitude and size. We then used these relations to map out the SDSS spectroscopic completeness as a function of intrinsic size, stellar mass, and redshift. In this appendix, we take a different tack: using the same empirical relations derived in Section 4.1, we consider the SDSS spectroscopic completeness for the specific galaxies in the vD08 sample (based on the sizes and masses given by vD08), if they were to be placed at low redshift.

The results of this exercise are shown in Figure 12. Each panel of this figure represents one of the three important spectroscopic selection criteria described in Section 4.1. In each panel, the larger points represent individual galaxies from the vD08 sample placed at $z=0.01,0.02, \ldots, 0.12$, the fine gray points show our main sample of massive, red sequence galaxies at $0.06<z<0.12$, and the hatched region shows where objects are not selected as spectroscopic targets.

The first panel of this figure represents the star/galaxy separation criterion. Objects are only selected for spectroscopic follow-up where $\Delta_{\mathrm{SG}} \equiv m_{\mathrm{PSF}, r}-m_{\mathrm{mod}, r} \geqslant 0.3 \mathrm{mag}$. It is clear from this panel that most of the vD08 galaxies are bright and large enough to be clearly distinguished from stars for $z \lesssim 0.12$. That said, the two smallest and lowest mass galaxies from the sample would not satisfy the star/galaxy separation criterion if placed at $z \gtrsim 0.10$.
The second panel represents the $\left(m_{\mathrm{Pet}, r}>15, \Theta_{\mathrm{Pet}}<2^{\prime \prime}\right)$ fiber saturation selection criterion. While all of the $\mathrm{vD} 08$ galaxies would satisfy this selection, it is clear that the highest surface brightness galaxies in the sample come very close to this selection limit. This selection would therefore be important for galaxies only slightly smaller and/or brighter than the highest surface brightness galaxies in the vD08 sample.

Finally, the third panel represents the ( $m_{\mathrm{fib}, g}<15, m_{\mathrm{fib}, r}<$ $\left.15, m_{\mathrm{fib}, r}<14.5\right)$ criterion designed to avoid cross-talk between spectroscopic fibers. (Note that since all red sequence galaxies are red in $(g-r)$, the $m_{\mathrm{fib}, g}<15$ criterion is made redundant by the $m_{\mathrm{fib}, r}<15$ criterion. For this reason, we only plot $m_{\mathrm{fib}, r}$ and $m_{\mathrm{fib}, i}$ in this panel.) This is clearly the most important selection criterion for compact, massive galaxies: none of the vD08 galaxies would be selected as SDSS spectroscopic targets if placed at $z \lesssim 0.04$; conversely, for $z \gtrsim 0.06$, most would satisfy this selection criterion.

\section{REFERENCES}

Abazajian, K. N., et al. 2009, ApJS, 182, 543

Baldwin, J. A., Phillips, M. M., \& Terlevich, R. 1981, PASP, 93, 5

Bell, E. F., \& de Jong, R. S. 2001, ApJ, 550, 212

Bell, E. F., McIntosh, D. H., Katz, N., \& Weiberg, M. D. 2003, ApJS, 149, 289 Bell, E. F., Zheng, X. Z., Papovich, C., Borch, A., Wolf, C., \& Meisenheimer, K. 2007, ApJ, 663, 834

Bezanson, R., van Dokkum, P. G., Tal, T., Marchesini, D., Kriek, M., Franx, M., \& Coppi, P. 2009, ApJ, 697, 1290

Blanton, M. R., \& Roweis, S. 2007, AJ, 133, 734

Blanton, M. R., et al. 2005, AJ, 129, 2562

Brammer, G., et al. 2009, ApJ, 706, L173

Bruzual, G., \& Charlot, S. 2003, MNRAS, 344, 1000

Buitrago, F., Trujillo, I., Barro, G., Gallego, J., Zamorano, J., \& Conselice, C. J. 2008, ApJ, 687, L61

Cappellari, M., et al. 2009, ApJ, 704, L34

Cattaneo, A., Dekel, A., Faber, S. M., \& Guiderdoni, B. 2008, MNRAS, 389, 567

Cennaro, A. J., \& Trujillo, I. 2009, ApJ, 696, L43

Chabrier, G. 2003, ApJ, 586, L133

Cimatti, A., et al. 2004, Nature, 430, 184

Cimatti, A., et al. 2008, A\&A, 482, 21

Conroy, C., Gunn, J. E., \& White, M. 2009, ApJ, 699, 486

Croton, D. J., et al. 2006, MNRAS, 365, 11 
Cutri, R. M., et al. 2003, Explanatory Supplement to the 2MASS All Sky Data Release and Extended Mission Products, NASA/IPAC Infrared Science Archive, http://www.ipac.caltech.edu/2mass/releases/allsky/doc/

Daddi, E., et al. 2004, ApJ, 617, 746

Daddi, E., et al. 2005, ApJ, 626, 680

Damen, M., et al. 2009, ApJ, 690, 937

Damjanov, I., et al. 2009, ApJ, 695, 101 (D09)

Dekel, A., \& Birnboim, Y. 2006, MNRAS, 368, 2

De Lucia, G., Springel, V., White, S D. M., Croton, D., \& Kauffmann, G. 2006, MNRAS, 366, 499

Faber, S. M., et al. 2007, ApJ, 665, 265

Fan, L., Lapi, A., De Zotti, G., \& Danese, L. 2009, ApJ, 689, L101

Franx, M., van Dokkum, P. G., Fórster-Schreiber, N. M., Wuyts, S., Labbé, I., \& Toft, S. 2008, ApJ, 688, 770

Franx, M., et al. 2003, ApJ, 587, L79

Gallazzi, A., Charlot, S., Brinchmann, J., White, S D. M., \& Tremonti, C. A. 2005, MNRAS, 362, 41

Glazebrook, K., et al. 2004, Nature, 430, 181

Guo, Y., McIntosh, D. H., Mo, H. J., Katz, N., van den Bosch, F. C., Weinberg, M., Weinmann, S. M., Pasquali, A., \& Yang, X. 2009, MNRAS, 398, 1129

Hopkins, P. F., Bundy, K., Murray, N., Quataert, E., Lauer, T., \& Ma, C.-P. 2009, MNRAS, 398, 898

Juneau, S., et al. 2005, ApJ, 619, L135

Kauffmann, G., White, S D. M., Heckman, T., Ménard, B., Brinchmann, J., Charlot, S., Tremonti, C., \& Brinkmann, J. 2004, MNRAS, 353, 713

Kauffmann, G., et al. 2003a, MNRAS, 341, 33

Kauffmann, G., et al. 2003b, MNRAS, 346, 1055

Khochfar, S., \& Silk, J. 2009, MNRAS, 397, 506

Kriek, M., van Dokkum, P. G., Labb'e, I., Franx, M., Illingworth, G. D., Marchesini, D., \& Quadri, R. 2009, ApJ, 700, 221

Kriek, M., van der Wel, A., van Dokkum, P. G., Franx, M., \& Illingworth, G. D. 2008a, ApJ, 682, 896

Kriek, M., et al. 2006, ApJ, 649, L71

Kriek, M., et al. 2008b, ApJ, 677, 219

Longhetti, M., et al. 2007, MNRAS, 374, 614

Marchesini, D., van Dokkum, P. G., Förseter-Schreiber, N. M., Franx, M., Labbé, I., \& Wuyts, S. 2008, ApJ, 701, 1765

McCarthy, P. J. 2004, ARA\&A, 42, 477

McCarthy, P. J., et al. 2004, ApJ, 614, L9

Menci, N., Fontana, A., Giallongo, E., \& Salimbeni, S. 2005, ApJ, 632, 49
Muzzin, A., Marchesini, D., van Dokkum, P. G., Labb'e, I., Kriek, M., \& Franx M. 2009, ApJ, 701, 1839

Naab, T., Johansson, P. H., \& Ostriker, J. P. 2009, ApJ, 699, L178

Padmanabhan, N., et al. 2008, ApJ, 674, 1217

Park, C., Choi, Y.-Y., Vogeley, M. S., Gott, J. R., \& Blanton, M. R. 2007, ApJ, 658, 898

Peng, C. Y., Ho, L. C., Impey, C. D., \& Rix, H.-W. 2002, AJ, 124, 266

Saracco, P., Longhetti, M., \& Andreon, S. 2009, MNRAS, 392, 718

Shankar, S., \& Bernardi, M. 2009, MNRAS, 396, L76

Shen, S., et al. 2003, MNRAS, 343, 978

Skrutskie, M. F., et al. 2006, AJ, 131, 1163

Stoughton, C., et al. 2002, AJ, 123, 485

Strauss, M. A., et al. 2002, AJ, 124, 1810

Taylor, E. N., et al. 2009, ApJS, 183, 295

Thakar, A. R., Szalay, A., Fekete, G., \& Gray, J. 2008, Comput. Sci. Eng., 10, 30

Toft, S., et al. 2007, ApJ, 671, 285

Trujillo, I., Cenarro, A. J., de Lorenzo-Cáceres, A., Vazdekis, A., de la Rosa, I. G., \& Cava, A. 2009, ApJ, 692, L118

Trujillo, I., Conselice, C., Bundy, K., Cooper, M. C., Eisenhardt, P., \& Ellis, R. C. 2007, MNRAS, 382, 109

Trujillo, I., et al. 2006, ApJ, 650, 18

Valentinuzzi, T., et al. 2010, ApJ, 712, 266

van der Wel, A., Bell, E. F., van den Bosch, F. C., Gallazzi, A., \& Rix, H.-W 2009, ApJ, 698, 1232

van der Wel, A., Franx, M., Wuyts, S., van Dokkum, P. G., Huang, J., Rix, H.-W., \& Illingworth, G. D. 2006, ApJ, 652, 97

van der Wel, A., Holden, B., Zirm, A. W., Franx, M., Rettura, A., Illingworth, G. D., \& Ford, H. C. 2008, ApJ, 688, 48

van Dokkum, P. G., et al. 2006, ApJ, 638, 59

van Dokkum, P. G., Kriek, M., \& Franx, M. 2009, Nature, 460, 717

van Dokkum, P. G., et al. 2008, ApJ, 677, L5 (vD08)

Weinmann, S. M., Kauffmann, G., van den Bosch, F. C., Pasquali, A., McIntosh, D. H., Mo, H., Yang, X., \& Guo, Y. 2009, MNRAS, 394, 1213

Williams, R. J., Quadri, R. F., Franx, M., van Dokkum, P. G., \& Labb'e, I. 2009, ApJ, 691, 1879

York, D. G., et al. 2000, AJ, 120, 1579

Zheng, X. Z., Bell, E. F., Papovich, C., Wolf, C., Meisenhemier, K., Rix, H.-W., Rieke, G. H., \& Sommerville, R. 2007, ApJL, 661, 41

Zirm, A. W., et al. 2007, ApJ, 656, 66 